\title{
AGN black hole mass estimates using polarization in broad emission lines
}

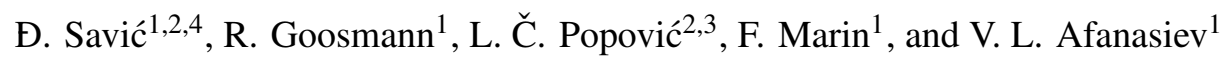 \\ 1 Observatoire Astronomique de Strasbourg, Université de Strasbourg, CNRS, UMR 7550, 11 rue de l'Université, 67000 Strasbourg, \\ France \\ 2 Astronomical Observatory Belgrade, Volgina 7, 11060 Belgrade, Serbia \\ e-mail: djsavic@aob.rs \\ 3 Department of Astronomy, Faculty of Mathematics, University of Belgrade, Studentski trg 16, 11000 Belgrade, Serbia \\ 4 Astrophysical Observatory of the Russian Academy of Sciences, Nizhnij Arkhyz, Karachaevo-Cherkesia 369167, Russia
}

Received 1 November 2017 / Accepted 6 January 2018

\begin{abstract}
Context. The innermost regions in active galactic nuclei (AGNs) have not yet been spatially resolved, but spectropolarimetry can provide insight into their hidden physics and geometry. From spectropolarimetric observations in broad emission lines and assuming equatorial scattering as a dominant polarization mechanism, it is possible to estimate the mass of supermassive black holes (SMBHs) residing at the center of AGNs.

Aims. We explore the possibilities and limits, and put constraints on the method for determining SMBH masses using polarization in broad emission lines by providing more in-depth theoretical modeling.

Methods. We used the Monte Carlo radiative transfer code sтокEs to explore polarization properties of Type-1 AGNs. We modeled equatorial scattering using flared-disk geometry for a set of different SMBH masses assuming Thomson scattering. In addition to the Keplerian motion, which is assumed to be dominant in the broad-line region (BLR), we also considered cases of additional radial inflows and vertical outflows.

Results. We modeled the profiles of polarization plane position angle $\varphi$, degree of polarization, and total unpolarized lines for different BLR geometries and different SMBH masses. Our model confirms that the method can be widely used for Type-1 AGNs when viewing inclinations are between $25^{\circ}$ and $45^{\circ}$. We show that the distance between the BLR and scattering region (SR) has a significant impact on the mass estimates and the best mass estimates are when the SR is situated at a distance 1.5-2.5 times larger than the outer BLR radius.

Conclusions. Our models show that if Keplerian motion can be traced through the polarized line profile, then the direct estimation of the mass of the SMBH can be performed. When radial inflows or vertical outflows are present in the BLR, this method can still be applied if velocities of the inflow/outflow are less than $500 \mathrm{~km} \mathrm{~s}^{-1}$. We also find that models for NGC 4051, NGC 4151, 3C 273, and PG0844+349 are in good agreement with observations.
\end{abstract}

Key words. galaxies: active - quasars: supermassive black holes - polarization - scattering

\section{Introduction}

Active galactic nuclei (AGNs) are known to be among the most powerful and steady radiation sources in the Universe. The huge amount of energy is produced by the accretion of matter onto supermassive black holes (SMBHs, Lynden-Bell 1969) whose mass ranges from $10^{6}$ to $10^{10} M_{\odot}$ (Kormendy \& Richstone 1995). The energy released by the growth of the black hole exceeds the binding energy of the host galaxy bulge (Fabian 2012). Thus, we can expect that AGNs have a strong feedback on their environment, due to the strong interaction of the energy and radiation produced by accretion with the surrounding gas of the host galaxy. This can lead to heating or ejection of the interstellar gas, which can prematurely terminate star formation in the galaxy bulge. This is strongly supported by the observed correlation between the mass of the central SMBH with luminosity, stellar velocity dispersion $\sigma_{*}$, or bulge mass (Kormendy \& Ho 2013), which indicates that the there is a coevolution of the SMBHs and the host galaxies (Heckman \& Best 2014). Measuring SMBH masses is a crucial task in order to understand how they are linked with the evolution of galaxies and AGNs.
Our ability to estimate the masses of AGN central black holes has significantly advanced in recent years (see, e.g., Peterson 2014, for a review). Several methods (both direct and indirect) have been developed. Direct methods are those for which the mass of the black hole is obtained from stellar dynamics by studying the motions of individual stars around the black hole (Genzel et al. 2010; Meyer et al. 2012) or gas dynamics (see, e.g., Miyoshi et al. 1995). Indirect methods use observables that are tightly correlated with black hole mass. One such example is $M_{\mathrm{bh}}-\sigma_{*}$ relation (Gebhardt et al. 2000; Ferrarese et al. 2001; Ferrarese \& Ford 2005). The most reliable (direct) mass measurements of SMBHs come from reverberation mapping (Blandford \& McKee 1982) of broad emission lines in about sixty AGNs (Bentz \& Katz 2015). However, reverberation mapping includes some unknown assumptions of Keplerian motion and photoionization as the dominant physical processes in the broad-line region (BLR).

A method of AGN black hole mass estimation using polarization in the broad lines given by Afanasiev \& Popović (2015, hereafter AP15), assumes that broad-line photons are emitted from the disk-like region undergoing Keplerian motion, after 
which they are scattered by the surrounding dusty torus, resulting in polarization in the broad emission lines. This method is in good agreement with the reverberation method and offers a number of advantages over traditional reverberation mapping. This method needs only one epoch of observations and does not use as much telescope time as the reverberation mapping method. It can be applied to lines from different spectral ranges, thus allowing black hole mass measurements for AGNs at different cosmological epochs (for more details, see Afanasiev \& Popović 2015). We note here that in this method the approximation of one scattering event per line photon was used, and that the contributions of multiple scattering events were not taken into account. Because the polarization is very sensitive to kinematics and geometrical setup (Goosmann \& Gaskell 2007), the full treatment of 3D radiative transfer with polarization is required to test this method. The aim of this work is to explore the AP15 method applying more accurate radiative transfer modeling. First we modeled the polarization in the broad lines using the sToKEs code, and then we compared the calculated polarization with observed in four Type-1 AGNs.

The paper is organized as followed. In Sect. 2 we give the description of the method for mass determination using polarization in broad lines, in Sect. 3 we describe parameters used for models, in Sect. 4 we give basic information on the observed objects used here. Our results are given in Sect. 5. In Sect. 6 we discuss our results and in Sect. 7 we briefly outline the main conclusions.

\section{AP15 method}

According to the unified model(Antonucci 1993; Urry \& Padovani 1995), every AGN hosts an accreting SMBH surrounded by a dusty torus along the equatorial plane. When the line of sight towards the central engine is unobscured, permitted broad spectral lines are prominent in the optical spectra in Type-1 objects. Broad lines are emitted from the broad-line region (BLR), highdensity clouds $\left(\sim 10^{10} \mathrm{~cm}^{-3}\right)$ situated around an accreting black hole with a global covering factor on the order of 0.1 (Netzer 2013). We can expect near Keplerian motion of the emitting gas in the BLR (Gaskell 2009). Farther away, the central region is surrounded by a geometrically thick toroidal structure of gas and dust with large radial optical depth (Krolik \& Begelman 1988). The inner side of the torus is directly illuminated and one can expect an abundance of free electrons in this part.

The BLR is surrounded by a co-planar scattering region (SR) that produces polarized broad lines, and a characteristic change in polarization plane position angle $\varphi$ across the line profile can be expected (Smith et al. 2005). According to AP15, $\varphi$ in the broad emission line is affected by the velocity field in the BLR and has a specific linear relationship between $\log V$ and $\log (\tan \varphi)$. As shown in Fig. 1 (right), if we have separation in the velocity field, it will affect $\varphi$ across the line.

Let us briefly describe the method. If the motion in the BLR is Keplerian, for the projected velocity in the plane of the scattering region, we can write (see Afanasiev et al. 2014, AP15)

$V_{i}=V_{i}^{\mathrm{rot}} \cos \theta=\sqrt{\frac{G M_{\mathrm{BH}}}{R_{i}}} \cos \theta$,

where $V_{i}$ is the rotational velocity of emitting gas, $M_{\mathrm{BH}}$ is the $\mathrm{BH}$ mass, $R_{i}$ is the distance from the center of the disk, $G$ is the gravitational constant, and $\theta$ is the angle between the disk and the plane of scattering (Afanasiev \& Popović 2015); $R_{i}$ can be connected with the corresponding polarization angle $\varphi_{i}$ as $R_{i}=R_{\mathrm{sc}} \tan \varphi_{i}$, where $R_{\mathrm{sc}}$ is the distance from the center to the SR (Fig. 1). When we substitute this into Eq. (1) taking into account the contribution of the different parts of the disk, the velocity-angle dependence can be transformed as

$\log \frac{V_{i}}{c}=a-0.5 \log \left(\tan \left(\varphi_{i}\right)\right)$

where $c$ is the speed of light. The expected relation between velocity and $\varphi$ is shown in Fig. 1 (right). The constant $a$ is related to the black hole mass as

$a=0.5 \log \frac{G M_{\mathrm{BH}} \cos ^{2} \theta}{c^{2} R_{\mathrm{sc}}}$.

In the case of a thin SR (equatorial scattering region), a good approximation would be to take $\theta \sim 0$. In this case, the relation between velocities and $\varphi$ does not depend on the inclination since the BLR is emitting a nearly edge-on oriented line light to the SR. From the previous equation, the mass of the black hole can be calculated as

$M_{\mathrm{BH}}=1.78 \times 10^{2 a+10} \frac{R_{\mathrm{sc}}}{\cos ^{2} \theta} M_{\odot} \approx 1.78 \times 10^{2 a+10} R_{\mathrm{sc}}\left[M_{\odot}\right]$,

or

$\log \frac{M_{\mathrm{BH}}}{M_{\odot}}=(10+2 a) \log \left(1.78 R_{\mathrm{sc}}\right)$,

where $R_{\mathrm{sc}}$ is in light days.

\section{Simulation of equatorial scattering}

\subsection{Radiative transfer code}

We used the radiative transfer code stokEs (Goosmann \& Gaskell 2007; Marin et al. 2012, 2015) to investigate polarization in the broad emission line in AGNs. It is a 3D radiative transfer code based on the Monte Carlo approach. It follows single photons from their creation inside the emitting region through processes such as electron or dust scattering until they become absorbed or until they manage to reach a distant observer. Initially, it was developed to study the ultraviolet (UV) and optical continuum polarization induced by electron and dust scattering in the radio-quiet AGNs, but it is suitable for studying many astrophysical objects of various geometries (Marin \& Goosmann 2014). We used the latest 1.2 version of the code stokes, which is publicly available ${ }^{1}$.

\subsection{Parameters of the model}

In our model, a point-like continuum source is situated in the center, emitting isotropic unpolarized radiation for which the flux is given by a power-law spectrum $F_{C} \propto v^{-\alpha}$ with $\alpha=2$. Since we are investigating the spectral range around a specific line, the chosen value for the spectal index $\alpha=2$ will not affect our research.

The continuum source is surrounded by a BLR which is finally surrounded by a SR. The BLR and SR are modeled using

1 http://wwW. stokes-program.info/ 

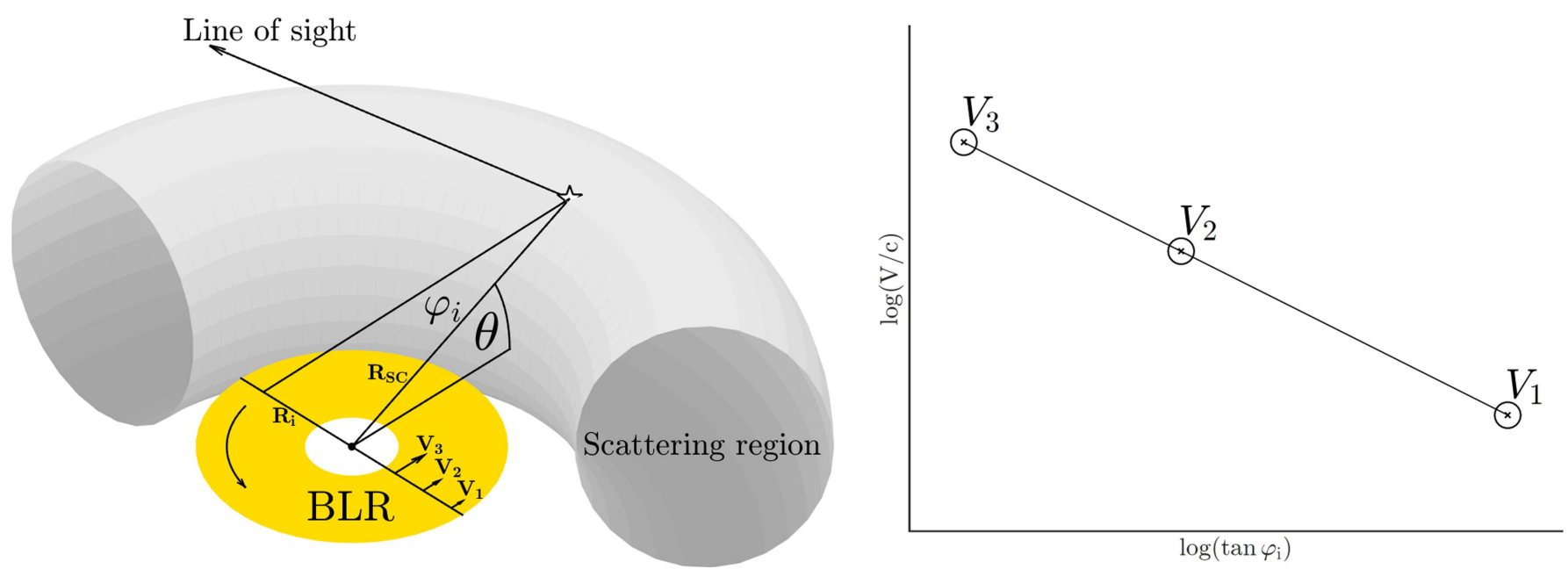

Fig. 1. Schematic view of light being scattered from the inner part of the torus (left). Expected relation between $\varphi$ and velocity intensity (right).

flared-disk geometry with a half-opening angle from the equatorial plane of $15^{\circ}$ (covering factor $\sim 0.1$ ) for the BLR and $35^{\circ}$ for the SR. A high covering factor for the SR is necessary in order to obtain the observed profile of the $\varphi$. A low covering factor of the SR gives a very small amplitude in the $\varphi$ profile. For the BLR inner radius $R_{\text {in }}^{\mathrm{BLR}}$, we adopted the value obtained by reverberation mapping (Kaspi et al. 2005; Bentz et al. 2006, 2013). The BLR outer radius was set by dust sublimation (predicted by Netzer \& Laor 1993)

$R_{\mathrm{out}}^{\mathrm{BLR}}=0.2 L_{\mathrm{bol}, 46}^{0.5}$,

where $L_{\mathrm{bol}, 46}$ is the bolometric luminosity given in $10^{46} \mathrm{ergs} \mathrm{s}^{-1}$. Bolometric luminosity is approximated from optical nuclear luminosity (Runnoe et al. 2012)

$\log L_{\text {iso }}=4.89+0.91 \log L_{5100}$,

where $\log L_{5100}$ is the optical nuclear luminosity. After correcting for average viewing angle, we obtain $\log L_{\text {bol }}=0.75 \log L_{\text {iso }}$. In our model, the BLR is transparent to photons, i.e., photons can freely travel from the inner side to the outer side of the BLR. This is in good agreement if BLR is perceived as a clumpy medium with small filling factor. For the flattened BLR (Gaskell 2009)

$v_{\text {Kepler }}>v_{\text {turb }} \gtrsim v_{\text {inflow }}$,

where $v_{\text {Kepler }}$ is Keplerian velocity, $v_{\text {turb }}$ is the turbulence velocity, and $v_{\text {inflow }}$ is the inflow velocity.

In our model, SR is a radially thin region as we assume that the light is being scattered dominantly by free electrons (Thomson scattering) in the innermost part of the torus. We assume that the electron density is decreasing radially outwards in the form of the power law $n_{\mathrm{e}} \propto r^{-1}$. The $\mathrm{SR}$ inner radius $R_{\mathrm{in}}^{\mathrm{SR}}$ is found from the IR reverberation mapping (Kishimoto et al. 2011; Koshida et al. 2014). The SR outer radius $R_{\text {out }}^{\text {SR }}$ was chosen such that the BLR half-opening angle when viewed from the edge of the SR is $25^{\circ}$. Investigations by Marin et al. (2012) for the SR with the flared-disk geometry have shown that optically thin SR $(\tau \leq 0.1)$ cannot produce sufficient polarization for Type-1 viewing angles. On the contrary, for optical depths $\tau>3$ multiple scattering can occur, resulting in depolarization. For this reason, we chose to set the total optical depth in radial direction to be $\tau=1$. An illustration of the model is shown in Fig. 2.

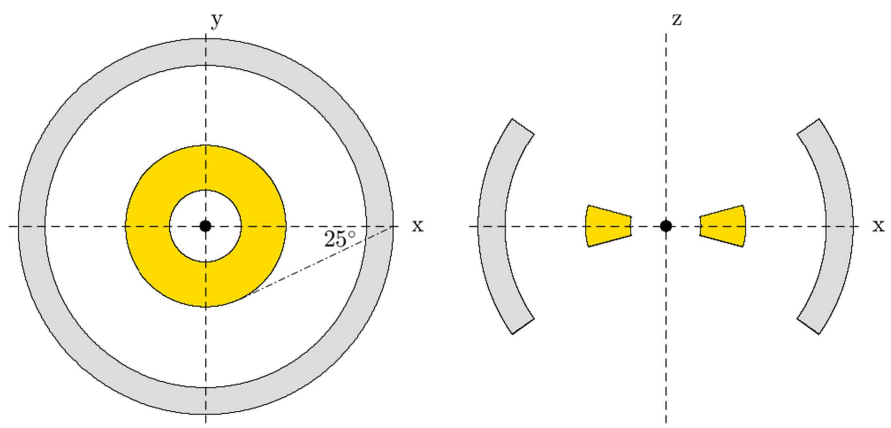

Fig. 2. Model geometry of the BLR (yellow) and the scattering disk (gray) in the face-on (left) and edge-on (right) views.

\subsection{Generic models}

We generated four probe models for which the central SMBH has a mass of $10^{6}, 10^{7}, 10^{8}$, and $10^{9} M_{\odot}$. We expect that the BLR distance from the center increases when the mass of the central SMBH increases, since the mass of the SMBH scales very well with the luminosity of the AGN (Laor 2000; Gu et al. 2001). In order to determine the size and the position of the BLR, as well as the SR for our probe models, we compiled 14 AGNs for which $\mathrm{BH}$ masses and inner radii of the BLR and SR are known from reverberation mapping (see Table 1). form

We fitted the $M_{\mathrm{bh}}$-radius relation with a power law in the

$\log M_{\mathrm{BH}}=C_{1} \log R+C_{2}$,

where $R$ takes the values for $R_{\text {in }}^{\mathrm{BLR}}, R_{\text {out }}^{\mathrm{BLR}}$, and $R_{\text {in }}^{\mathrm{SR}}$. In Fig. 3, we show the mass-radius relationship with $1 \sigma$ uncertainty. Fit constants are listed in Table 2. This way we obtained a rough estimate of the BLR and SR sizes for our model setup. We represent the goodness of fit using the adjusted coefficient of determination $\bar{R}^{2}$.

With known fit constants, we generated values for the $R_{\mathrm{in}}^{\mathrm{BLR}}, R_{\mathrm{out}}^{\mathrm{BLR}}, R_{\mathrm{in}}^{\mathrm{SR}}$, and $R_{\mathrm{out}}^{\mathrm{SR}}$ for the set of four different SMBHs (see Table 3). Our approach is the following. For each model with known input mass of the SMBH, we solve $3 \mathrm{D}$ radiative transfer using stoKes; we then apply the AP15 method to the simulated results; and finally we compare the value of the obtained SMBH mass with the value of input SMBH mass. 
Table 1. List of objects with known $\log M_{\mathrm{bh}}, L_{5100}, R_{\mathrm{in}}^{\mathrm{BLR}}$ and $R_{\mathrm{in}}^{\mathrm{SR}}$ that we used for models.

\begin{tabular}{lcccccccc}
\hline \hline Object & $z$ & $\begin{array}{c}\log M_{\text {bh }} \\
M_{\odot}\end{array}$ & $\log L_{5100}$ & $\begin{array}{c}\text { Ref. } \\
\mathrm{erg} \mathrm{s}^{-1}\end{array}$ & $R_{\text {in }}^{\text {BLR }}$ & $\begin{array}{c}\text { Ref. } \\
\text { Light days }\end{array}$ & $\begin{array}{c}R_{\text {in }}^{\text {SR }} \\
\text { Light days }\end{array}$ \\
\hline Mrk335 & 0.02579 & 7.230111 & $43.71 \pm 0.06$ & 1 & $14.10 \pm 1.20$ & 3,4 & 141.7 & 7 \\
Mrk590 & 0.02639 & 7.569731 & $43.42 \pm 0.07$ & 1 & $25.50 \pm 6.00$ & 3 & 34.7 & 7 \\
Ark120 & 0.03271 & 8.068022 & $43.78 \pm 0.07$ & 1 & $32.70 \pm 3.00$ & 3 & 428.8 & 7,8 \\
Mrk79 & 0.02219 & 7.611875 & $43.61 \pm 0.04$ & 1 & $29.30 \pm 14.30$ & 3 & 69.5 & 7 \\
PG0844+349 & 0.06400 & 7.858308 & $44.24 \pm 0.04$ & 6 & $12.20 \pm 5.20$ & 3 & 104.3 & 7 \\
Mrk110 & 0.03529 & 7.292445 & $43.60 \pm 0.04$ & 1 & $26.90 \pm 7.00$ & 3 & 104.3 & 7 \\
NGC3227 & 0.00386 & 6.774994 & $42.24 \pm 0.11$ & 1 & $4.40 \pm 0.40$ & 3 & 25.0 & 7,8 \\
NGC3516 & 0.00884 & 7.394509 & $42.73 \pm 0.21$ & 1 & $14.60 \pm 1.30$ & 3 & 61.2 & 7 \\
NGC4051 & 0.00234 & 6.129727 & $41.96 \pm 0.20$ & 1 & $2.50 \pm 0.20$ & 3 & 38.0 & 7,8 \\
NGC4151 & 0.00332 & 7.555236 & $42.09 \pm 0.22$ & 1 & $6.00 \pm 0.40$ & 3 & 44.0 & 7,8 \\
3C273 & 0.15834 & 8.838866 & $45.90 \pm 0.02$ & 1 & $306.8 \pm 90.9$ & 3 & 963 & 8 \\
NGC4593 & 0.00900 & 6.882240 & $42.87 \pm 0.18$ & 1 & $4.50 \pm 0.65$ & 3 & 43.0 & 7 \\
NGC5548 & 0.01718 & 7.718341 & $43.23 \pm 0.10$ & 1,2 & $17.60 \pm 8.86$ & 3,5 & 60.0 & 7 \\
Mrk817 & 0.03146 & 7.586162 & $43.68 \pm 0.05$ & 1 & $21.20 \pm 14.70$ & 3 & 180.0 & 7 \\
PG1613+658 & 0.12900 & 8.338928 & $44.71 \pm 0.03$ & 1 & $35.00 \pm 15.10$ & 3,6 & 595.0 & 7 \\
PG1700+518 & 0.29200 & 8.785679 & $45.53 \pm 0.01$ & 1 & $251.80 \pm 42.35$ & 3,6 & 687.0 & 7 \\
\hline
\end{tabular}

Notes. Mass was estimated from reverberation mapping using the $\mathrm{H} \beta$ line and for $\langle f\rangle=4.3$ (Grier et al. 2013).

References. Optical luminosities are taken from (1) Bentz et al. (2013), (2) Peterson et al. (2013). The estimates for $R_{\text {in }}^{\text {BLR }}$ are taken from (3) Zu et al. (2011), (4) Grier et al. (2012), (5) Grier et al. (2013), (6) Kaspi et al. (2000). The estimates for $R_{\text {in }}^{\text {SR }}$ are taken from (7) Koshida et al. (2014), (8) Kishimoto et al. (2011).
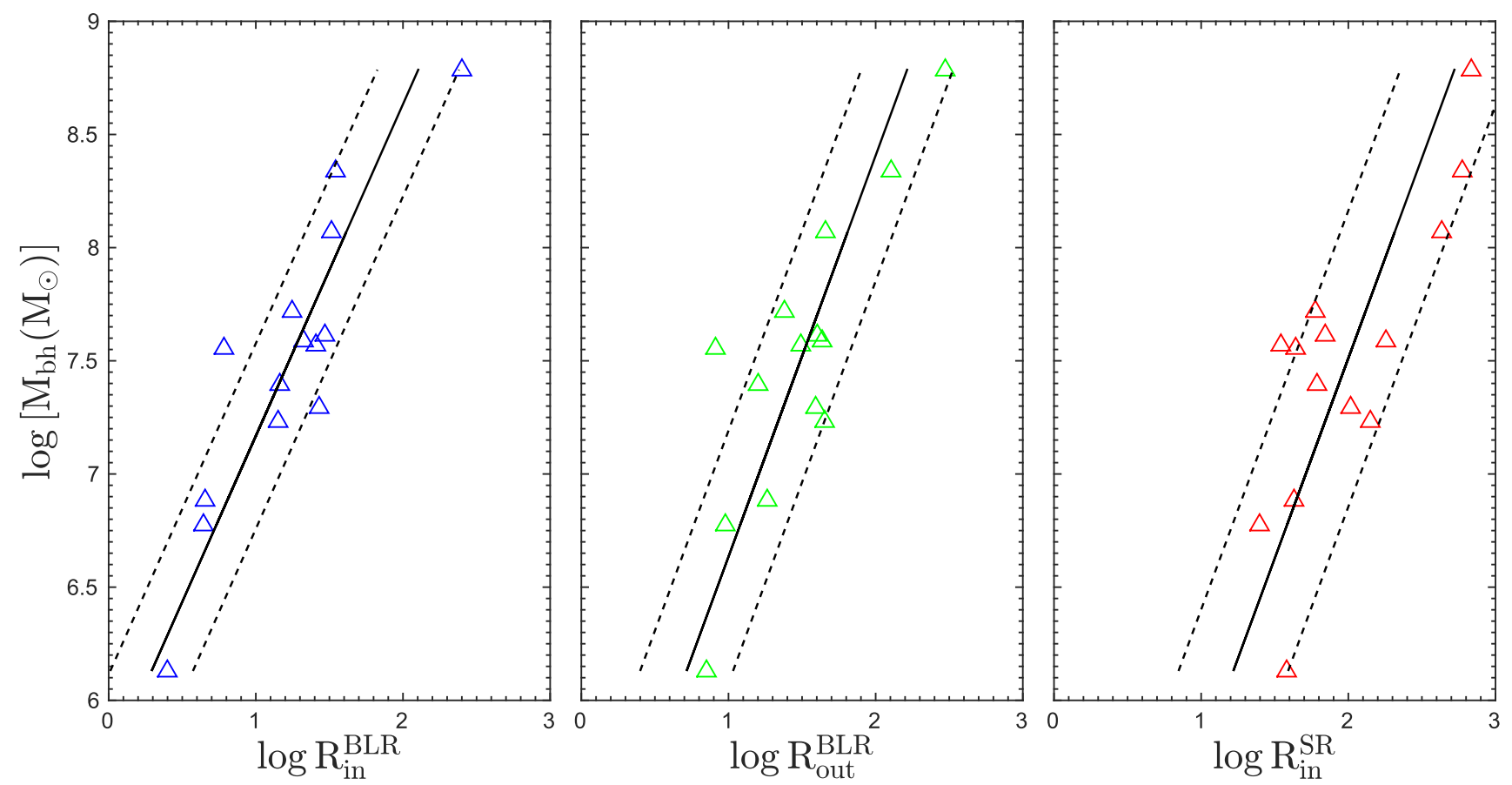

Fig. 3. Mass-radius relation, for $R_{\mathrm{in}}^{\mathrm{BLR}}(l e f t), R_{\mathrm{out}}^{\mathrm{BLR}}$ (middle), and $R_{\mathrm{in}}^{\mathrm{SR}}(r i g h t)$. Data taken from literature (see Table 1) are denoted as triangles; while solid lines represent the best fit. Dashed lines mark the $1 \sigma$ uncertainty.

\section{Observations}

We have selected four AGNs with prominent changes in $\varphi$ across the line profile: NGC4051, NGC4151, 3C273, and PG0844+349. Spectropolarimetry was done with the $6 \mathrm{~m}$ telescope of the Special Astrophysical Observatory of the Russian Academy of Sciences (SAO RAS) using a modified version of the SCORPIO spectrograph (see Afanasiev \& Moiseev 2005, 2011). Data reduction and the calculation of the polarization parameters, as well as corrections for the interstellar polarization, are done in the same way as described in Afanasiev \& Amirkhanyan (2012). Model parameters for these objects are given Table 4. In order to test the AP15 method theoretically, we modeled each of these objects using observational data available from the literature. This is important since we can perform direct comparison of the results obtained from the model with the newest spectropolarimetric observations using the SAO RAS $6 \mathrm{~m}$ telescope. The values of $R_{\mathrm{in}}^{\mathrm{BLR}}$ and $R_{\mathrm{in}}^{\mathrm{SR}}$ were taken from the 
Table 2. Constants $C_{1}$ and $C_{2}$ for the mass-radius relation (Eq. (8); Cols. 2 and 3).

\begin{tabular}{cccc}
\hline \hline$R$ & $C_{1}$ & $C_{2}$ & $\bar{R}^{2}$ \\
\hline$R_{\text {in }}^{\mathrm{BLR}}$ & $0.682 \pm 0.096$ & $-3.890 \pm 0.723$ & 0.7915 \\
$R_{\text {out }}^{\mathrm{BLR}}$ & $0.564 \pm 0.108$ & $-2.743 \pm 0.812$ & 0.6690 \\
$R_{\text {in }}^{\mathrm{SR}}$ & $0.566 \pm 0.127$ & $-2.248 \pm 0.958$ & 0.5899 \\
\hline
\end{tabular}

Notes. Adjusted coefficient of determination $\bar{R}^{2}$ (Col. 4) for the performed fit.

Table 3. Central SMBH masses, inner and outer radius of the BLR, and inner radius of the SR that we used in our model.

\begin{tabular}{|c|c|c|c|c|}
\hline Mass & $R_{\text {in }}^{\mathrm{BLR}}$ & $R_{\text {out }}^{\text {BLR }}$ & $R_{\text {in }}^{\mathrm{SR}}$ & $R_{\text {out }}^{\mathrm{SR}}$ \\
\hline$M_{\odot}$ & ld & ld & ld & ld \\
\hline $10^{6}$ & 1.597 & 4.385 & 13.968 & 20.262 \\
\hline $10^{7}$ & 7.681 & 16.076 & 51.372 & 74.277 \\
\hline $10^{8}$ & 36.944 & 58.934 & 188.939 & 272.288 \\
\hline $10^{9}$ & 177.700 & 216.043 & 694.893 & 998.170 \\
\hline
\end{tabular}

literature using the dust reverberation method (Kishimoto et al. 2011; Koshida et al. 2014, Table 1), while $R_{\text {out }}^{\text {BLR }}$ and $R_{\text {out }}^{\text {SR }}$ were computed in the same way as for the generic models. Model parameters for these objects are given in Table 4. Input mass was obtained by applying the AP15 method to the observational data.

NGC 4051 is a relatively nearby Seyfert 1 galaxy with cosmological redshift equal to 0.0023 , known for its highly variable X-ray flux (McHardy et al. 2004). NGC 4051 was extensively observed in the high-energy band to see if the rapid continuum variations observed in the X-ray spectra are correlated to the optical band fluctuations. This is not the case, even though the timeaveraged X-ray and optical continuum fluxes are well correlated. Only the flux of the broad $\mathrm{H} \beta$ line lags behind the optical continuum variations by 6 days, allowing us to estimate the mass of the central supermassive black hole (Peterson et al. 2000). The optical continuum polarization of NGC 4051 was measured by Martin et al. (1983) and Smith et al. (2002), who found a polarization degree of $0.52 \pm 0.09 \%$ and $0.55 \pm 0.04 \%$, respectively. The polarization position angle was found to be parallel to the radio axis of the AGN, such as expected for most Type-1 objects (Antonucci 1993).

$N G C 4151$ is a 1.5 Seyfert galaxy situated at $z=0.0033$ (de Vaucouleurs et al. 1991), which is sometimes considered to be the archetypal Seyfert 1 galaxy (Shapovalova et al. 2008, 2010). It is one of the brightest Type-1 AGNs in the X-ray and ultraviolet band, and its bolometric luminosity is on the order of $5 \times 10^{43} \mathrm{erg} \mathrm{s}^{-1}$ (Woo \& Urry 2002). The mass of its central supermassive black hole was estimated by optical and ultraviolet reverberation techniques and is estimated at $4.5 \times 10^{7} M_{\odot}$ (Bentz et al. 2006). Since NGC 4151 stands out, thanks to its high fluxes and proximity, its optical polarization has been extensively observed (see Marin et al. 2016). The averaged 4000$8000 \AA$ continuum polarization is below $1 \%$, with a polarization position angle parallel to the radio axis of the system. In the optical range, NGC 4151 shows flux variations of the continuum and of the broad lines up to a factor of ten or greater (Shapovalova et al. 2008, 2010). The wings of broad lines also vary greatly from very intensive ones corresponding to type Sy 1 in the maximum state of activity to almost complete absence in the minimum state of activity. In April 1984, the nucleus of NGC 4151 went through a very deep minimum, the broad wings of the hydrogen lines almost completely vanished, and the spectrum of the nucleus was identified as a Sy 2 (Penston \& Perez 1984). In this phase, the intensity in the broad component of a spectral line is too weak and the AP15 method probably could not be used. Another technique must be used to explore the geometry of the object, such as proved by Hutsemékers et al. (2017) and Marin (2017).

$3 C 273$ is a well-known flat-spectrum radio source quasar with broad emission lines. It is the brightest and one of nearest quasars known to us $(z=0.158$, Courvoisier et al. 1987, 1990). It is a radio-loud object, i.e., its radio to millimeter energy output is dominated by synchrotron emission from a kiloparsec, one-sided jet whose emission extends up to the infrared and optical bands. 3C 273 is particularly bright in the optical and ultraviolet domains, which enabled the detection of the polarization of the optical emission. Its mean optical core polarization was measured by Appenzeller (1968) and is on the order of $0.2 \pm 0.2 \%$, which is consistent with galactic interstellar polarization (Whiteoak 1966). The optical polarization emerging from the jet structure is high because they were resolved into a number of highly polarized knot structures by Thomson et al. (1993). Nevertheless, Balmer emission lines were first measured by Schmidt (1963) and allowed the central black hole mass to be determined from the average line profiles (Kaspi et al. 2000).

$P G 0844+349$ is a radio-quiet quasar at a cosmological redshift of $z=0.064$ that, unlike most quasars, was not originally detected in the radio frequency: at radio wavelengths, its nucleus is unresolved (Kellermann et al. 1994). It was first discovered in the Palomar Green sample (Schmidt \& Green 1983) and was found to possess strong Fe II emission and weak forbidden narrow lines, a behavior that is expected from narrowline Seyfert-1s (NLS1). On the other hand, the X-ray properties of PG $0844+349$ are aligned with the NLS1 classification (a steep soft X-ray spectrum and strong variability, see Boller 2001), and the optical polarization measurements achieved by Afanasiev et al. (2011) also point towards a regular NLS1 object (optical continuum polarization of $0.85 \pm 0.10 \%$ ). Hence, using Type-1 AGN reverberation mapping techniques, Peterson et al. (2004) estimated the mass of the central black hole to be $(9.24 \pm 3.81) \times 10^{8} M_{\odot}$.

\section{Results}

We present our results which can be divided into two parts: the results of modeling and a comparison of our models with the observations. The same convention used by Goosmann \& Gaskell (2007) was adopted in this work. Namely, $\varphi$ is parallel to the symmetry axis of the model when $\varphi=90^{\circ}$, which was observed for Type- 1 objects, or $\varphi$ is orthogonal to the symmetry axis when $\varphi=0^{\circ}$, which was observed for Type- 2 objects.

\subsection{Generic modeling}

We simulated different geometries of the BLR. We first performed the simulation for different masses of the black holes with assumption of a pure Keplerian motion, and then we considered the radial inflow and vertical outflow as additional components in gas motion to the Keplerian caused by the black hole mass. We simulated both cases where Keplerian motion is counterclockwise (positive) and clockwise (negative). 
Table 4. Central SMBH masses, inner and outer radius of the BLR and SR that we used in our model for comparison with the observed data.

\begin{tabular}{lccccc}
\hline \hline Object & $\log \left(M_{\mathrm{POL}} / M_{\odot}\right)$ & $\begin{array}{c}R_{\text {in }}^{\mathrm{BLR}} \\
\text { ld }\end{array}$ & $\begin{array}{c}R_{\text {out }}^{\mathrm{BLR}} \\
\text { ld }\end{array}$ & $\begin{array}{c}R_{\text {in }}^{\mathrm{SR}} \\
\text { ld }\end{array}$ & $\begin{array}{c}R_{\text {out }}^{\mathrm{SR}} \\
\text { ld }\end{array}$ \\
\hline NGC 4051 & $6.69 \pm 0.21$ & 4.3 & 15.0 & 38.1 & 53.7 \\
NGC 4151 & $7.21 \pm 0.27$ & 6.6 & 17.5 & 44.0 & 63.8 \\
3C 273 & $8.85 \pm 0.27$ & 306.8 & 440.6 & 963.7 & 2035.8 \\
PG0844+349 & $7.70 \pm 0.14$ & 12.2 & 77.4 & 189.0 & 357.6 \\
\hline
\end{tabular}
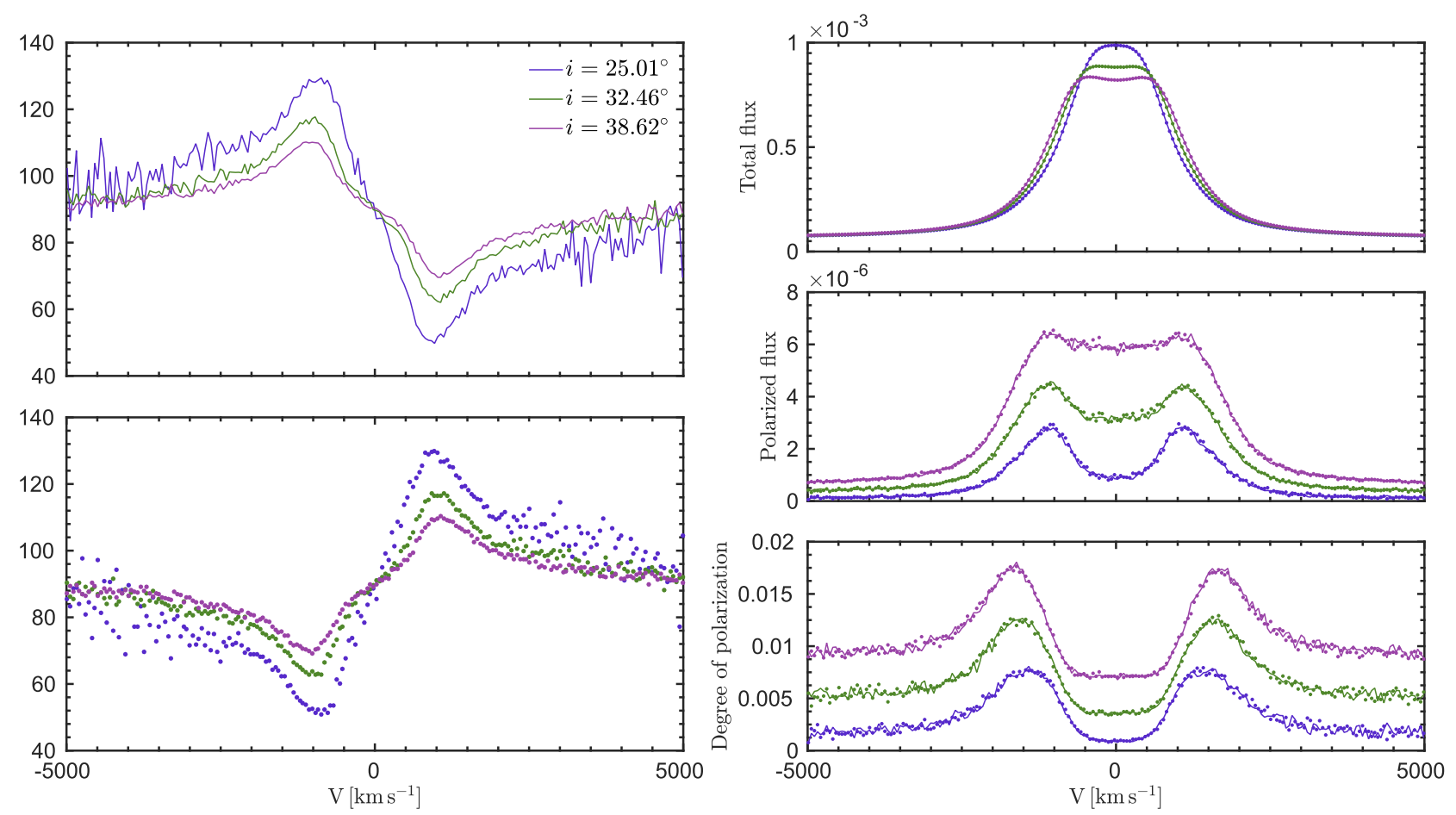

Fig. 4. Left panels: modeled polarization plane position angle $\varphi$ when the system is rotating counterclockwise (top) or when rotating clockwise (bottom). Right panels: total unpolarized flux (TF, top), polarized flux (PF, middle), degree of polarization (PO, bottom). The SMBH has a mass of $10^{6} M_{\odot}$. We plot the results in solid lines for three viewing inclinations: $i=25.01^{\circ}, 32.46^{\circ}$, and $38.62^{\circ}$, while dotted lines represent the results for the opposite direction of rotation. We note the symmetry of $\varphi$ with respect to the continuum level, due to the opposite direction of rotation. An opposite direction of rotation does not affect TF, PF, and PO. Total and polarized fluxes are given in arbitrary units.

\subsubsection{Pure Keplerian gas motion in the BLR}

We consider the pure Keplerian motion of the BLR emitting gas, taking that there are no other effects (e.g., outflows and inflows). We present the results of the four probe models. In Figs. 4 and 5, we show the simulated profiles of $\varphi$, polarized flux (PF), degree of polarization (PO), and total flux (TF) across the broad-line profile. Each scattering element can see the velocity resolved BLR emission which produces polarized lines that are broader than the unpolarized lines. The simulated degree of polarization is of the same order of magnitude as that obtained from the observations and is typically around $1 \%$ or less (Marin et al. 2016). From our models (Figs. 4 and 5, bottom right panels), we can see that the degree of polarization is sensitive to inclination. Extensive modeling with complex radiation reprocessing (see, e.g., Marin et al. 2012, for more details) have shown that the total PO is increasing as we start looking from the face-on viewing angle towards Type-2 viewing angles. Although we included only equatorial scattering in our model, the dependence of PO on inclination follows this trend. The PO profile peaks in the line wings and reaches a minimum in the line core, as was shown by Smith et al. (2005). This feature was very well observed for the case of Mrk 6 (Smith et al. 2002; Afanasiev et al. 2014) and it supports the suggested scattering geometry.

The polarization plane position angle is aligned with the disk rotation axis, hence also with the radio jet axis. In Figs. 4 and 5 (left panels), we show the simulated profiles of $\varphi$ for three viewing inclinations. The $\varphi$ profiles show a typical symmetric swing that was predicted for Type- 1 objects where the radiation from the Keplerian rotating disk-like BLR is being scattered by the outer dusty torus (Smith et al. 2005, AP15). The direction of rotation only affects $\varphi$, while TF, PF, and PO remain unaffected. For counterclockwise rotation, $\varphi$ reaches a maximum value in the blue part of the line and minimum in the red part of the line. The $\varphi$ swing occurs around the level of continuum $\varphi_{\mathrm{c}}=90^{\circ}$. Due to the symmetry of the model (also for all other models performed in the paper), $\varphi$ is symmetric with respect to the continuum polarization in such a way that for a given inclination $i$, it satisfies the following:

$\varphi\left(180^{\circ}-i\right)=180^{\circ}-\varphi(i)$. 

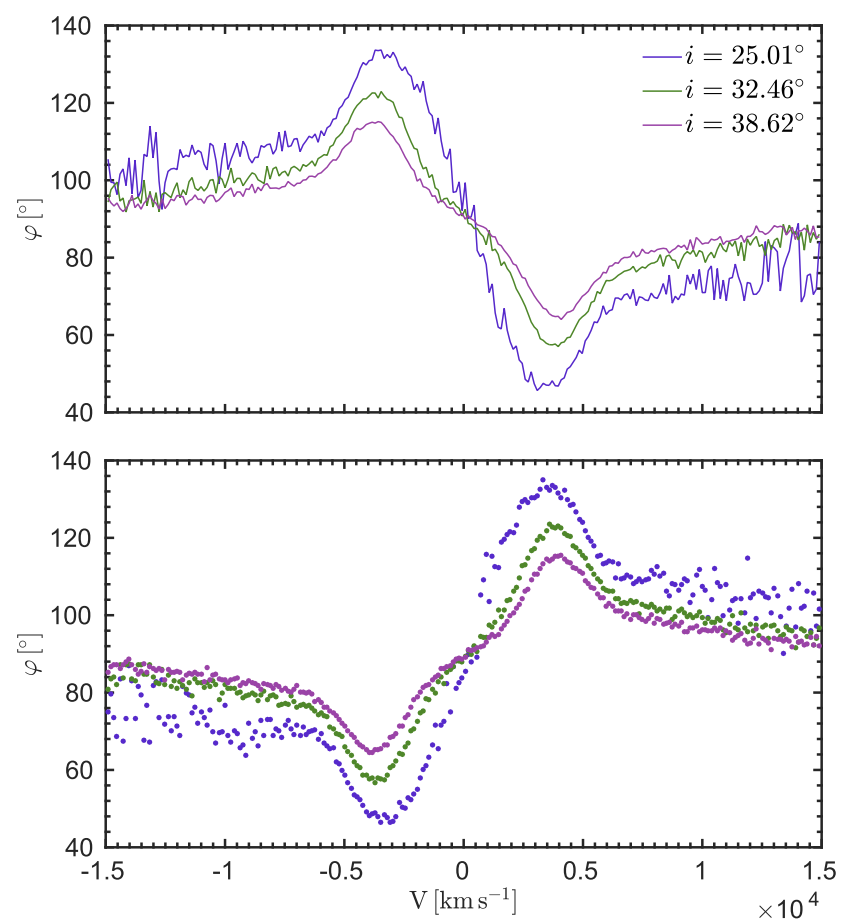

Fig. 5. Same as Fig. 4, but for SMBH of $10^{9} M_{\odot}$.

In other words, for a given half-opening angle of the torus $\theta_{0}$, and for Type- 1 inclinations where $0 \leq i \leq 90^{\circ}-\theta_{0}$, the observer can see one way of rotation, and the corresponding $\varphi$ profile will be as shown in Figs. 4 and 5. If the system is viewed for the Type-1 viewing angle where $90^{\circ}+\theta_{0} \leq i \leq 180^{\circ}$, the opposite direction of rotation is observed and the resulting $\varphi$ satisfies Eq. (9). This symmetry can be seen in Figs. 4 and 5 (left panels). Thus, spectropolarimetric observations of Type-1 Seyferts can disentangle the rotation direction of the gas by observing the $\varphi$ profile. Equation 9 is satisfied within the Monte Carlo uncertainty and it was used to improve photon statistics in our simulations by a factor of 2 by simply taking the average value of the Stokes parameters for inclinations $i$ and $180^{\circ}-i$.

When applying the AP15 method to the modeled data, we needed to consider polarization only in the broad line; thus, it was necessary to subtract the continuum polarization for all Type-1 inclinations:

$\Delta \varphi=\varphi-90^{\circ}$.

Since all of our observed objects are rotating clockwise (see Sect. 5.2), we performed the AP15 method assuming the opposite direction of rotation without introducing new simulations. In Fig. 6 (lower panels), we show the fit described by the AP15 method. We find that Keplerian motion can be traced across the $\varphi$ profile for Type- 1 viewing inclinations. The region inside the $1 \sigma$ error around the linear fit becomes smaller as we go from faceon towards edge-on inclinations. For inclinations $25^{\circ}$ or lower, the simulated data show much higher scatter around the straight line rather than for the cases with an average inclination.

The effect of a wide SR (in our case $\theta_{0}=35^{\circ}$ ) can lead to mass estimates that are a maximum of $\sim 1.5$ higher than those obtained for equatorial scattering, and only if the SR lies much farther away from the BLR (see Eq. (4)). In this case, the influence of the viewing inclination must be taken into account.

It is important to note that in Eq. (4), we used the inner radius of the SR in order to estimate the mass of the SMBH. However,
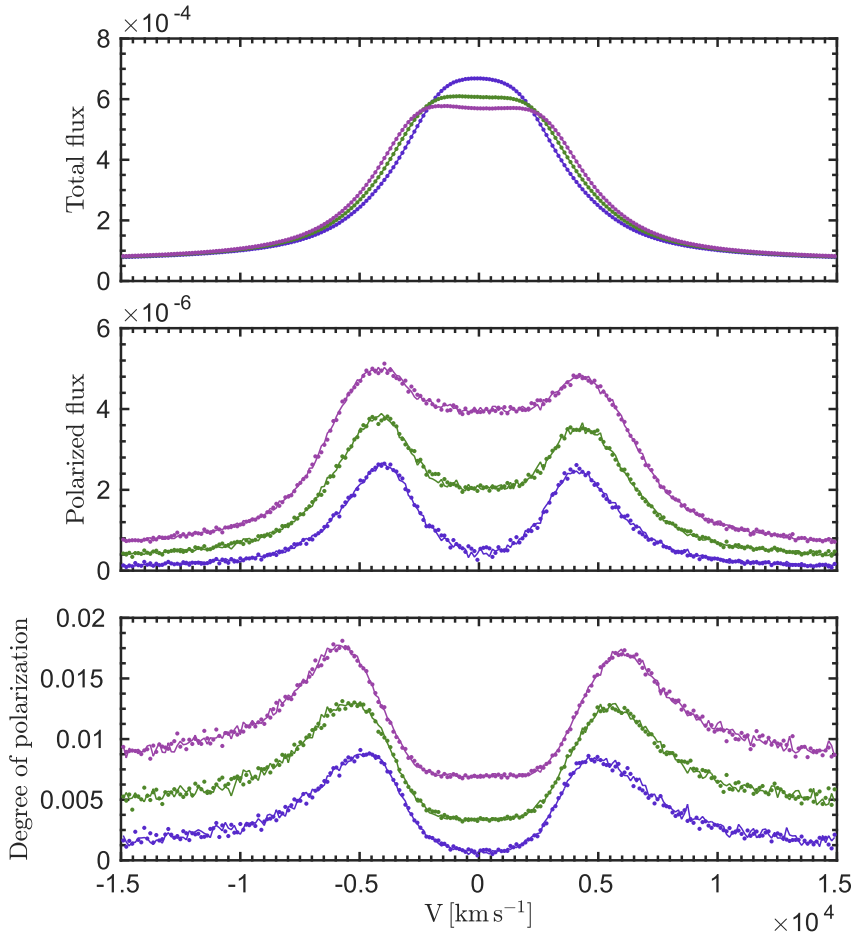

Table 5. Input mass (Col. 1), viewing inclinations (Col. 2), and masses obtained for probe models (Col. 3).

\begin{tabular}{ccc}
\hline \hline $\log M_{\text {input }}$ & $i\left(^{\circ}\right)$ & $\log \left(M_{\mathrm{MOD}} / M_{\odot}\right)$ \\
\hline \multirow{3}{*}{6} & 25.01 & $6.72 \pm 0.10$ \\
& 32.46 & $6.44 \pm 0.06$ \\
& 38.62 & $6.28 \pm 0.05$ \\
7 & 25.01 & $7.59 \pm 0.10$ \\
& 32.46 & $7.30 \pm 0.07$ \\
& 38.62 & $7.17 \pm 0.04$ \\
8 & 25.01 & $8.65 \pm 0.11$ \\
& 32.46 & $8.39 \pm 0.07$ \\
& 38.62 & $8.23 \pm 0.06$ \\
9 & 25.01 & $9.67 \pm 0.16$ \\
& 32.46 & $9.43 \pm 0.12$ \\
& 38.62 & $9.27 \pm 0.10$ \\
\hline
\end{tabular}

Notes. Masses are given in $M_{\odot}$.

the SR does not act as a mirror from which the light is being scattered from the inner wall. Scattering events occur in the entire SR, and they all contribute to the total $\varphi$ shape. Obtaining the value for parameter $a$ is a straightforward procedure, but the final estimated SMBH value largely depends on the actual value of $R_{\mathrm{sc}}$. In the optically thick media, the largest fraction of photons is scattered from the inner side of the SR.

One of the factors that has significant impact on the $\varphi$ amplitude is the mutual distance between the BLR and the SR (Smith et al. 2005). The amplitude of $\varphi$ decreases when mutual distance increases, which affects black hole mass estimation. Therefore, we investigated different cases with various mutual distances between the BLR and SR, while keeping the same thickness and optical depth as the SR. In Figs. 7 and 8, we show the influence of different mutual distance between the two regions, and how it affects the parameter $a$ and SMBH estimates. 

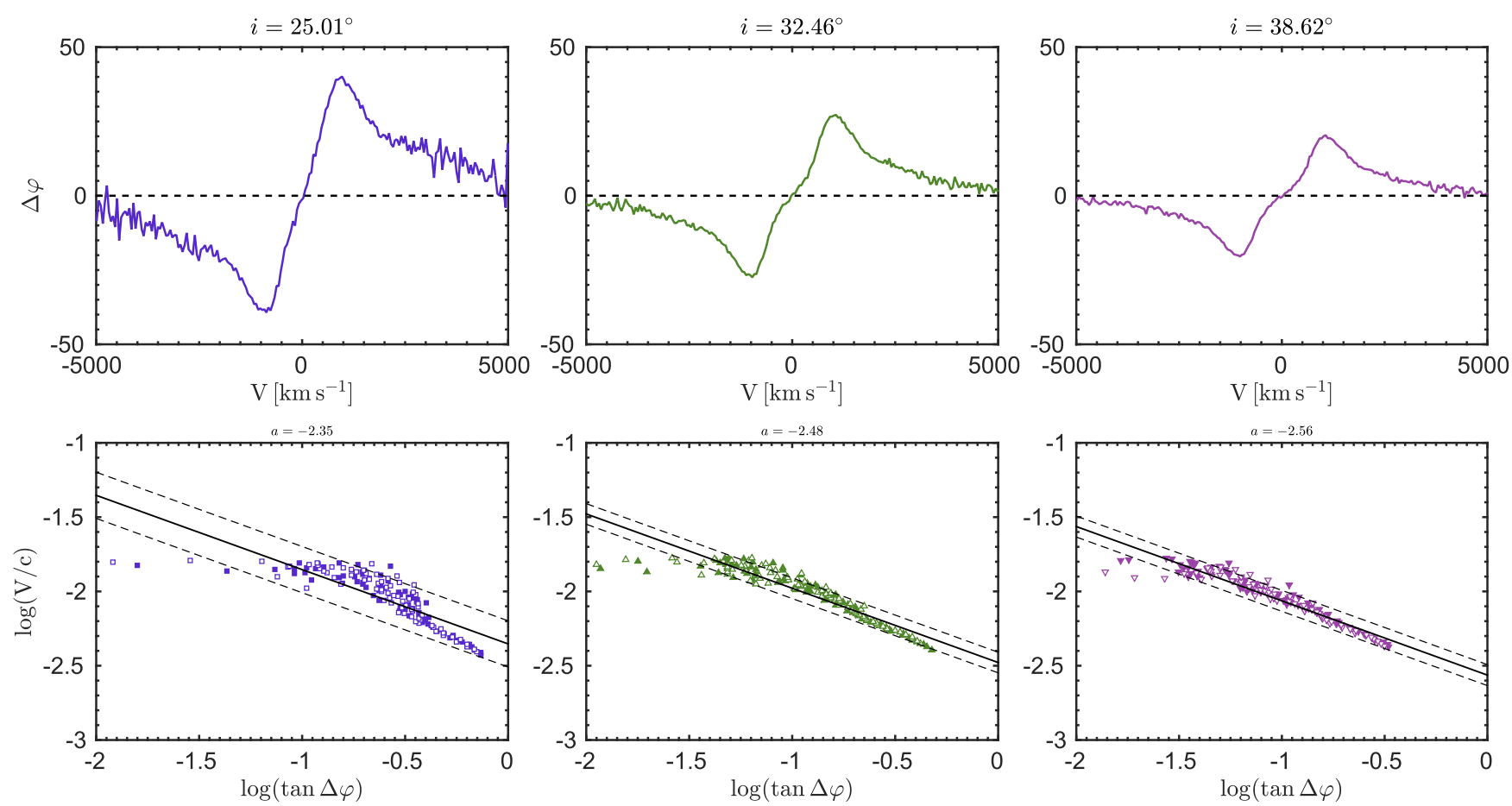

Fig. 6. Modeled polarization $\varphi$ (upper panels) and velocities (lower panels) across $\mathrm{H} \alpha$ profiles for the model with central mass of $10^{6} M_{\odot}$. Filled symbols are for the blue part of the line and open symbols are for the red part of the line. The solid line represents the best fit.

Our models show that the mutual distance between the BLR and SR has a great influence on the parameter $a$, which consequently greatly affects our black hole mass estimates. Parameter $a$ shows the same profile and the same inclination dependence for all simulated cases. Only when the SR is adjacent to the BLR do we obtain inclination independence of the SMBH mass estimates. Due to the nature of Eq. (4), SMBH mass estimates increase when the mutual distance increases. For a given accuracy of $10 \%$, we find that the best SMBH estimates for all four cases are when the ratio of the inner radius of SR and the outer radius of the BLR is between 1.5 and 2.5 (Fig. 8). For the inclinations of $25^{\circ}$ or less (face-on view), the contribution of equatorial scattering is low and we find that Keplerian motion cannot be recovered from the $\varphi$ profile.

\subsubsection{Keplerian motion and radial inflow}

We investigated a particular case when the BLR is undergoing a constant radial inflow. We tested three cases with BLR radial inflow velocity equal to $500 \mathrm{~km} \mathrm{~s}^{-1}, 1000 \mathrm{~km} \mathrm{~s}^{-1}$, and $2000 \mathrm{~km} \mathrm{~s}^{-1}$. As before, in Fig. 9 we show simulated profiles of $\varphi, \mathrm{PF}, \mathrm{PO}$, and TF. In this regime, the SR can see an additional component of the BLR velocity, which as a net effect increases the absolute value of the radial velocity that a single scattering element can see. This leads to additional line broadening (Fig. 9, lower right panel) when compared to the case with pure gas Keplerian motion only. As a resulting effect, the distance between the positions of the maximum and the minimum of the $\varphi$ is increased (Fig. 9, left panels). Therefore, for a low-velocity radial inflow, mass estimates of the SMBHs are slightly higher than those obtained in the case with Keplerian motion alone. This overestimation of the SMBH mass mostly affects the model for which the SMBH has a mass of $10^{6} M_{\odot}$. For the other models, Keplerian motion is even more dominant (except for the very extreme cases which are not expected) and the influence of the radial inflow can be neglected.

\subsubsection{Keplerian motion and vertical outflow}

Another contribution to velocity might be due to vertical outflows. We tested three cases for which the innermost one-third of the BLR is undergoing a constant vertical outflow of $500 \mathrm{~km} \mathrm{~s}^{-1}$, $1000 \mathrm{~km} \mathrm{~s}^{-1}$, and $2000 \mathrm{~km} \mathrm{~s}^{-1}$. In this case, the equatorial scattering elements will not see this velocity component. Scattering elements above the equatorial plane will see this component multiplied by a factor of $\cos \alpha$, where $\alpha$ is latitude, to a maximum of $\cos 35^{\circ}$. This can be neglected when the outflow velocity is much lower than Keplerian velocity. In Fig. 10, we show the results of simulated $\varphi, \mathrm{PF}, \mathrm{PO}$, and TF influenced by vertical outflows in the BLR of $2000 \mathrm{~km} \mathrm{~s}^{-1}$ for the case where SMBH has a mass of $10^{6} M_{\odot}$. The unpolarized line (bottom right panel) is additionally broadened in the wings. The polarized line (upper right panel) is almost the same as the one for the case with Keplerian motion only (Fig. 4, upper right panel), for the reasons explained above. Contribution of outflow velocity is highest for the nearly face-on view. In Fig. 11, left panels, the $\varphi$ profile shows an additional bump, which prevents us from correctly using the AP15 method. We would like to point out again that in our model, the BLR is transparent and that the observer can see the radiation coming from the approaching and the receding part of the BLR outflows. We know from observations that this is not the case (e.g., Mrk 6; Afanasiev et al. 2014), and we expect to observe radiation from the approaching side of the BLR outflows, while the radiation from the receding side of the BLR outflows should be blocked, thus affecting only the blue part of the line.

\subsection{Comparison with observations}

Here we present the fits of our model with observations in order to estimate the SMBH mass. We fit model data to observational data and compare the results, The results are given in Table 6, and below we discuss the results and visual comparison for each object. 
Đ. Savić et al.: AGN black hole mass estimates using polarization in broad emission lines
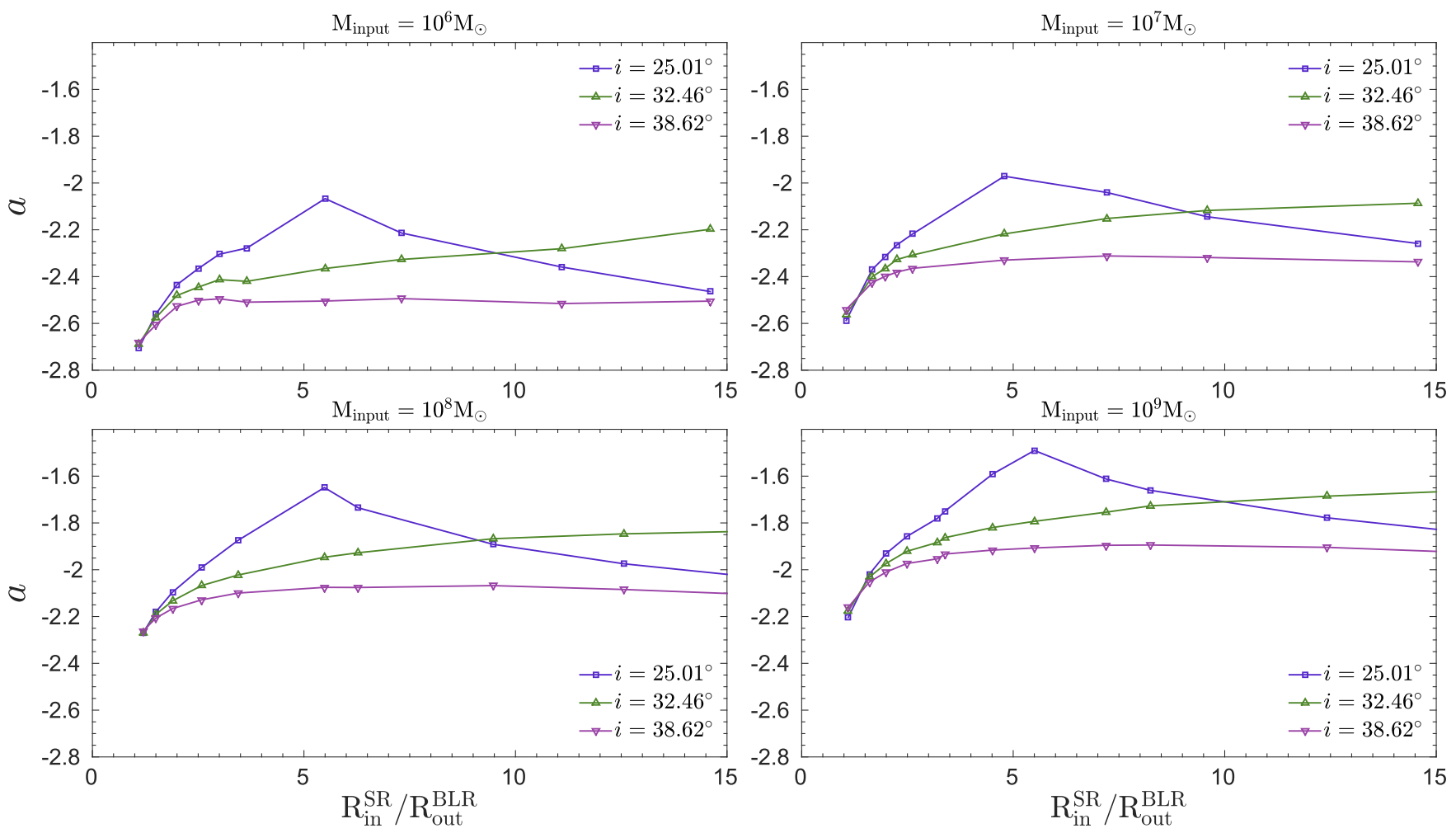

Fig. 7. Dependence of the parameter $a$ on the ratio between the inner radius of the $\mathrm{SR}\left(R_{\mathrm{in}}^{\mathrm{SR}}\right)$ and the outer radius of the BLR $\left(R_{\mathrm{out}}^{\mathrm{BLR}}\right)$ for three given inclinations.
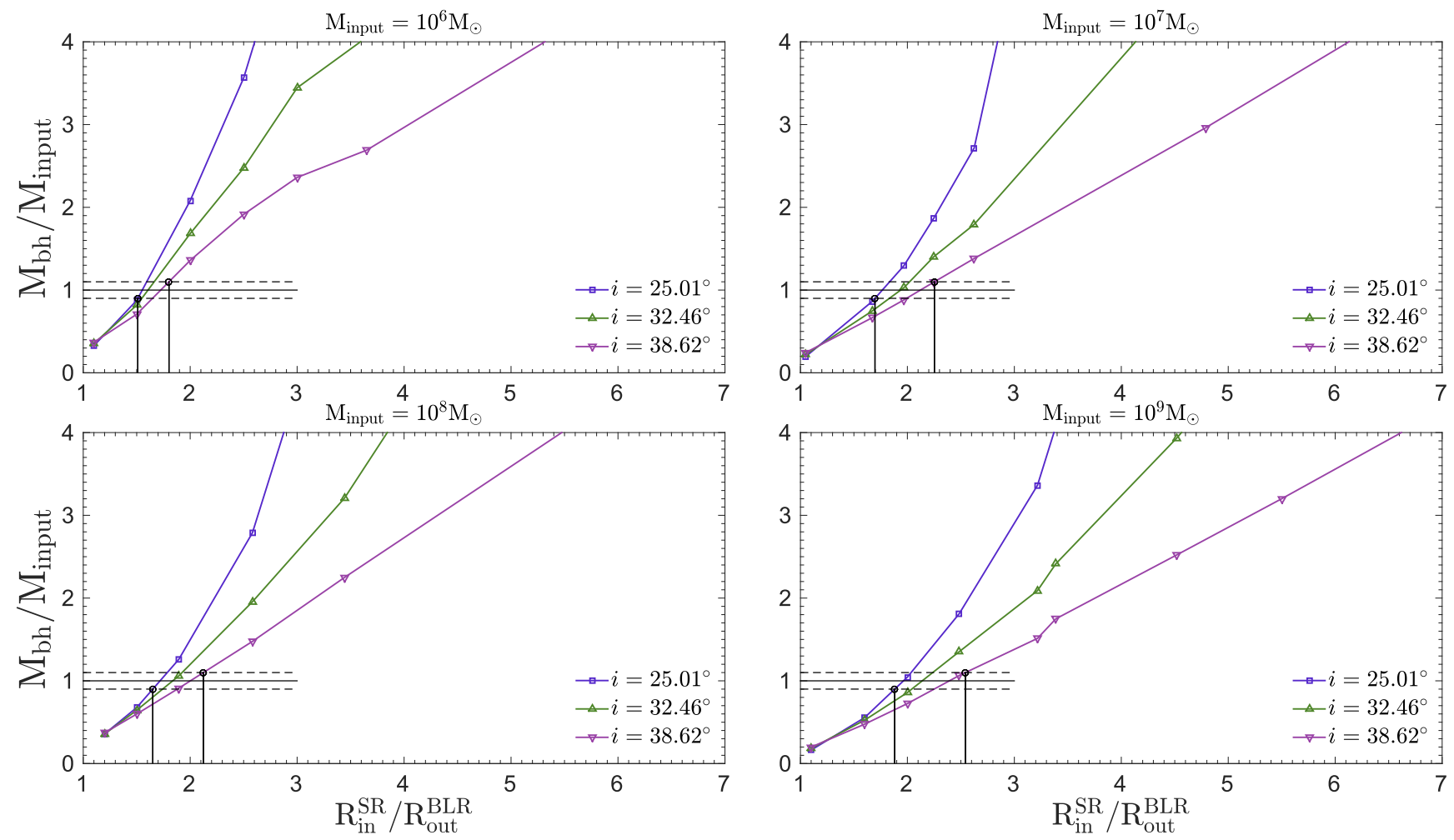

Fig. 8. Black hole mass estimation as a function of the ratio between the inner radius of the SR $\left(R_{\mathrm{in}}^{\mathrm{SR}}\right)$ and the outer radius of the BLR $\left(R_{\mathrm{out}}^{\mathrm{BLR}}\right)$ for three given inclinations. Horizontal dashed lines represent the interval of $10 \%$ deviation from the input mass (solid line). 

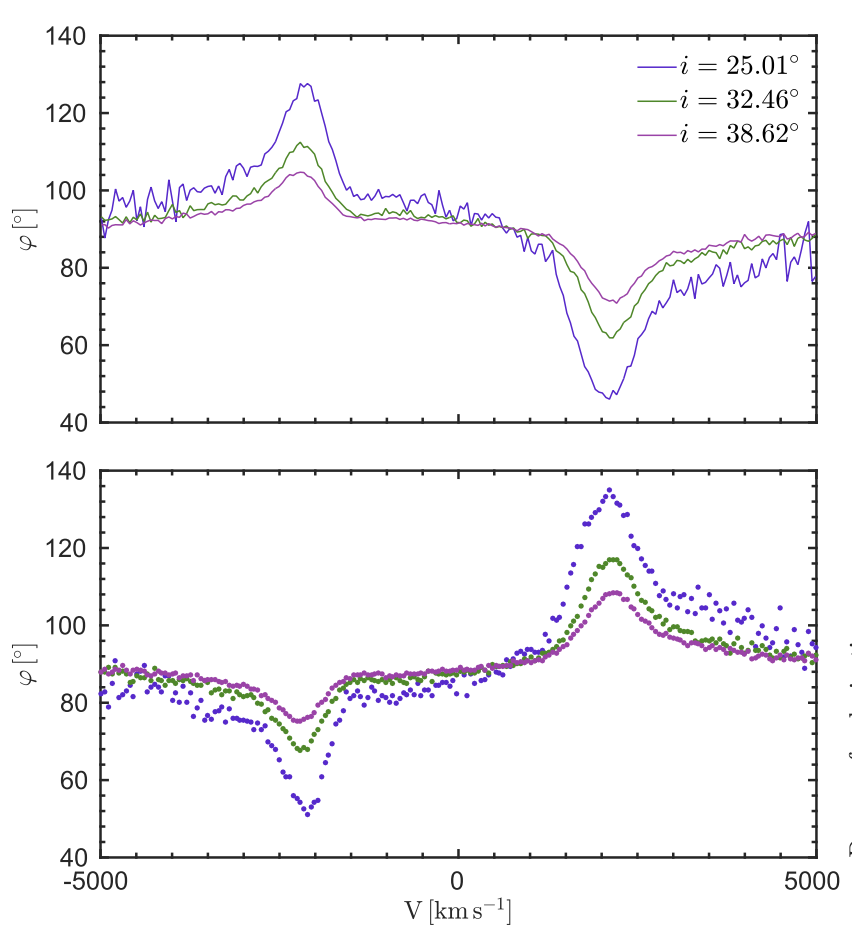
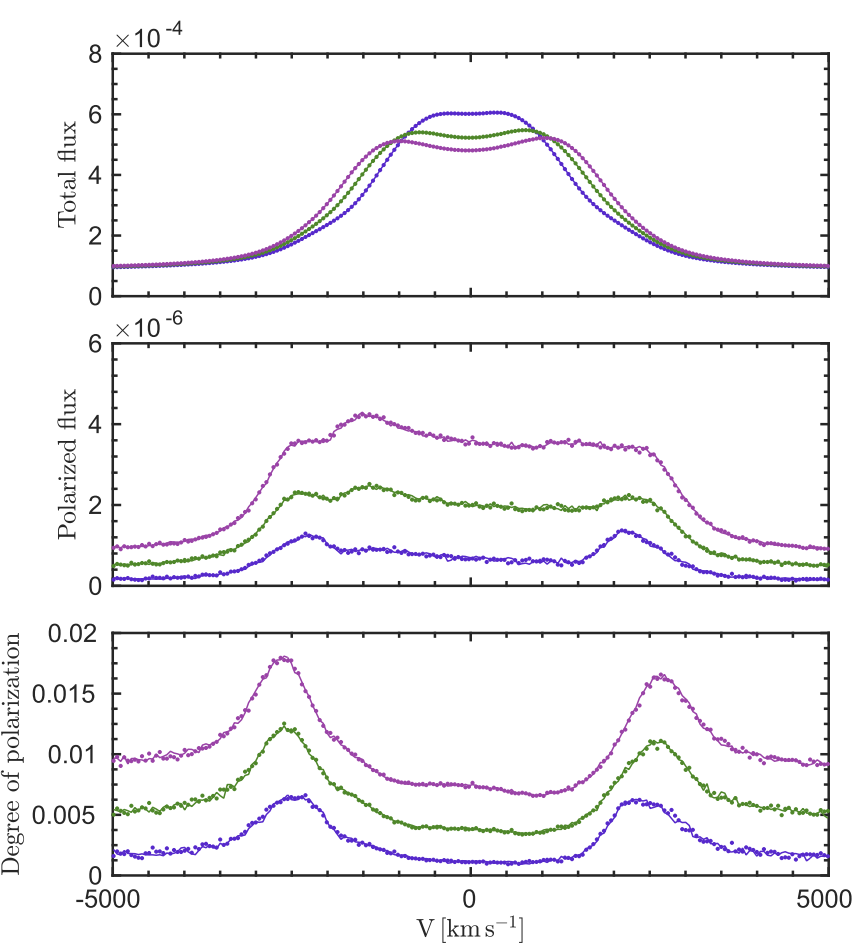

Fig. 9. Same as Fig. 4, but in addition to Keplerian motion, a large inflow of $2000 \mathrm{~km} \mathrm{~s}^{-1}$ is included in the BLR kinematics.
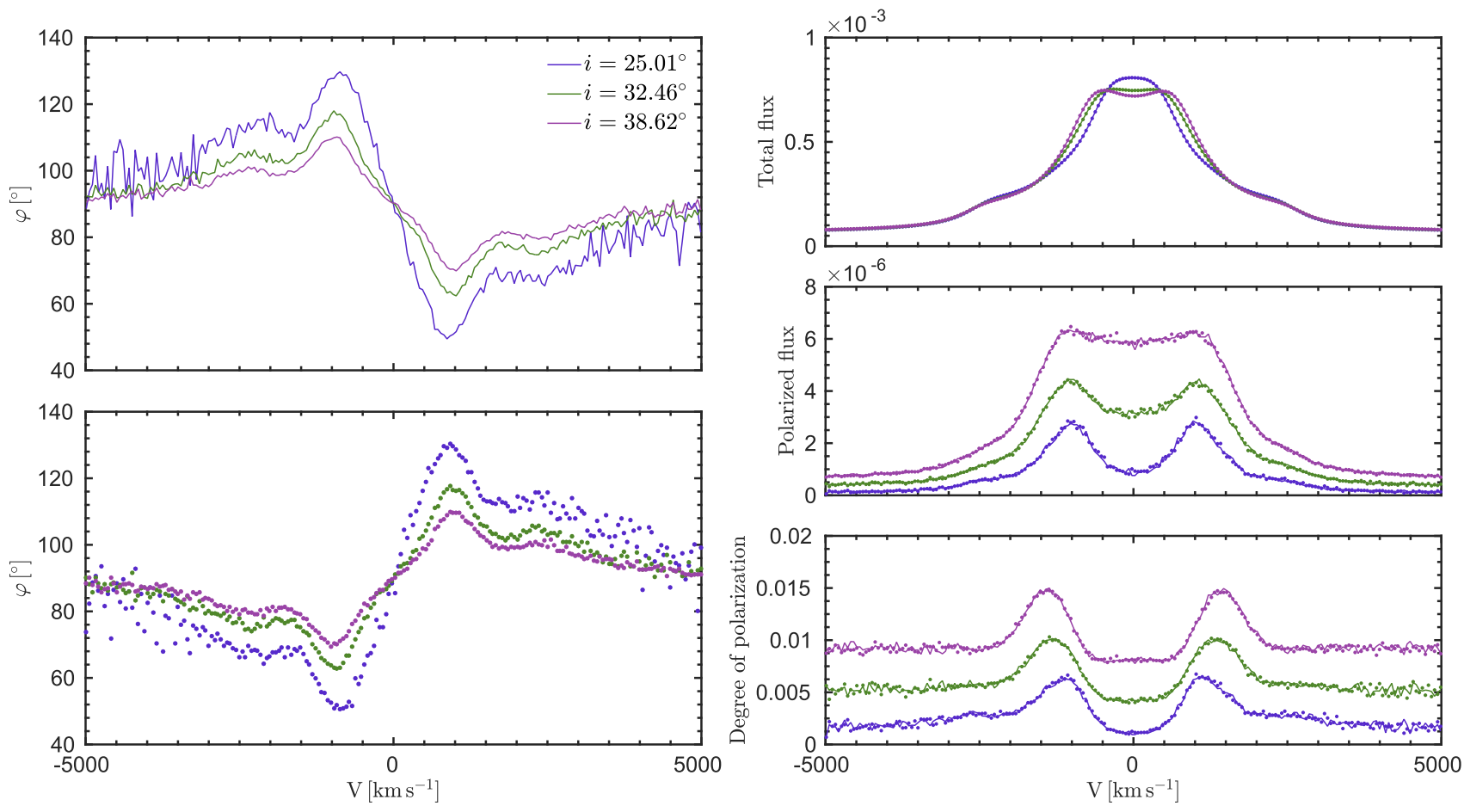

Fig. 10. Same as Fig. 4, except that here the inner one-third of the BLR undergoes a constant vertical outflow of $2000 \mathrm{~km} \mathrm{~s}^{-1}$.

$N G C 4051$ - We were able to obtain the expected $\varphi$ shape (Fig. 12, upper panels).

The amplitude of the $\varphi$ in the model is very close to the observed value for the lowest inclination for which $i=25.01^{\circ}$. For this inclination, the position of the maximum and the minimum of $\varphi$ is displaced, which yields the highest mass estimate. As we start viewing from higher inclinations, the $\varphi$ amplitude decreases and we are able to better fit the line wings (Fig. 12, lower panels), and the difference between the estimated values of the SMBH masses and the input mass is smaller. We can see that simulated data in the line wings for $i=25^{\circ}$ deviate from the theoretically predicted straight line. For intermediate inclinations the $1 \sigma$ offset is smaller and the fit is better.

Similarly, SMBH masses estimated from the fitting of the model data are higher than the input mass, as in the previous case. For this object, $R_{\text {in }}^{\mathrm{SR}} / R_{\text {out }}^{\mathrm{BLR}} \approx 2.54$.

$N G C 4151$ - Similarly to the previous case, we obtain the highest mass estimate for the lowest inclination. Keplerian motion is 
Đ. Savić et al.: AGN black hole mass estimates using polarization in broad emission lines
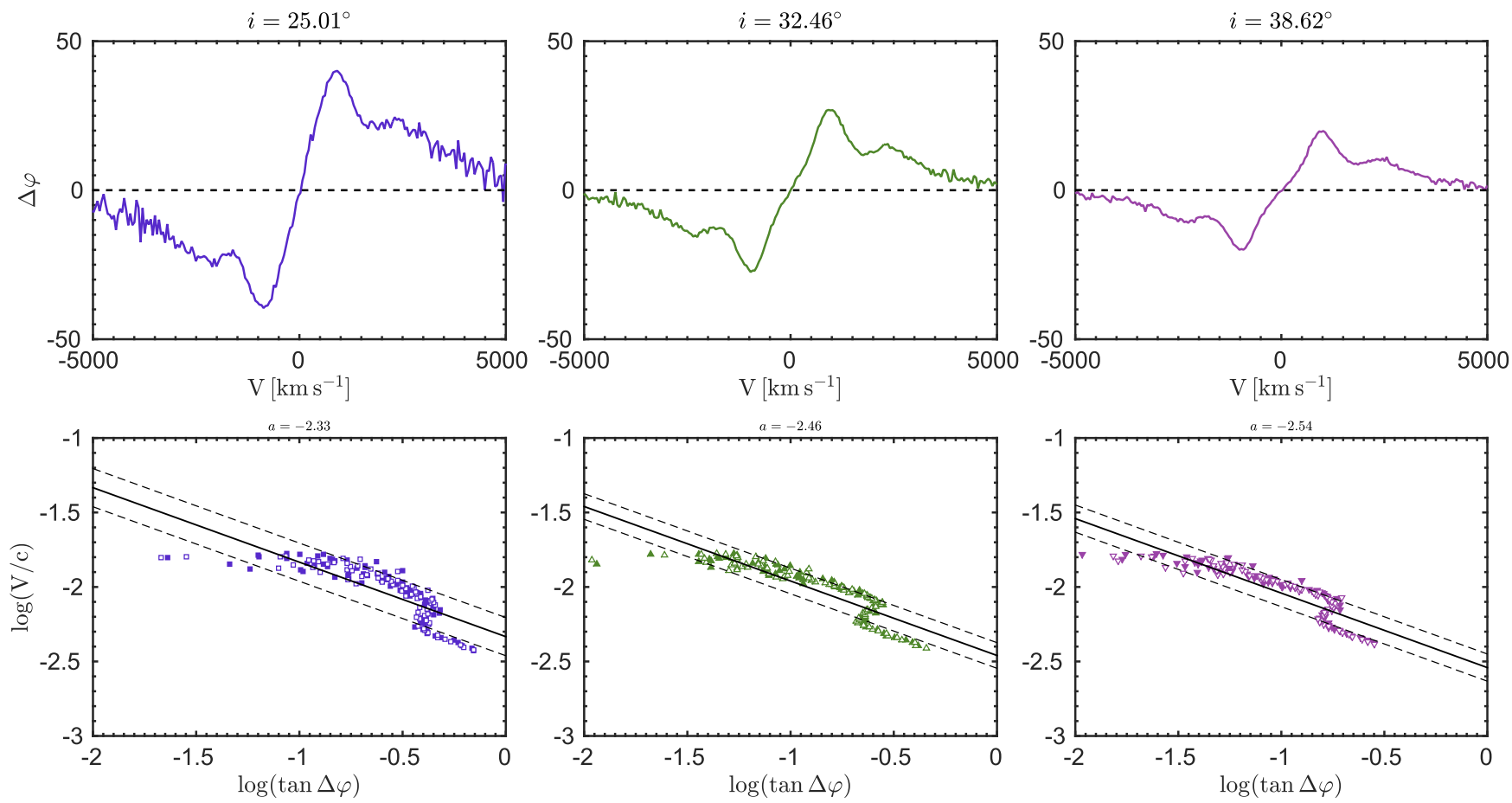

Fig. 11. Same as Fig. 6, except that here the inner one-third of the BLR undergoes a constant vertical outflow of $2000 \mathrm{~km} \mathrm{~s}^{-1}$.
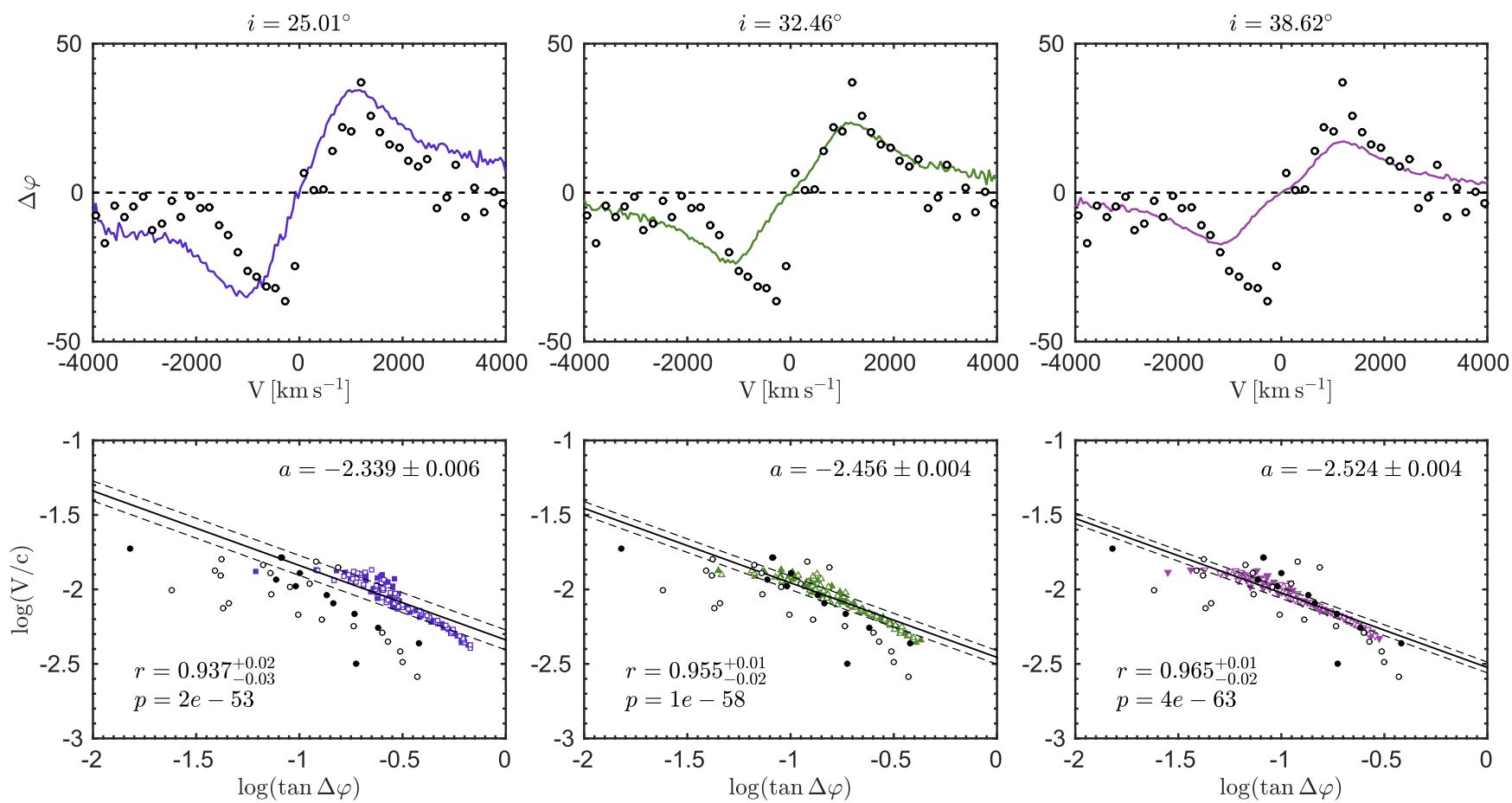

Fig. 12. Modeled broad-line polarization position angle $\varphi$ (upper panels) and velocities (lower panels) across H $\alpha$ profiles for NGC 4051 . In the upper panels, data obtained by the models are depicted as a line, while observed data are depicted by empty circles. In the lower panels, filled symbols are for the blue part of the line and the open symbols are for the red part of the line for model data. Black circles depict observed data. The solid black line represents the best fit. Values of the parameter $a$, correlation coefficient $r$, and the corresponding $p$ values are shown.

shown very well as a straight line (Fig. A.1, lower panels), where the $1 \sigma$ error is small, especially in the case where $i=39^{\circ}$. For this object, $R_{\text {in }}^{\mathrm{SR}} / R_{\text {out }}^{\mathrm{BLR}} \approx 2.51$.

We can see that in the extreme wings of the line, the modeled $\varphi$ becomes very sensitive to spectral resolution and this sensitivity is lower for higher inclinations.
$3 C 273$ - We obtained very low $\varphi$ dependence on inclination; however, the $\varphi$ amplitude is much smaller, around $19^{\circ}$ for all inclinations (Fig. A.2). Model data show a deviation from the straight line in the line wings; however, the scatter is much smaller than it is for the observational data. The ratio $R_{\mathrm{in}}^{\mathrm{SR}} / R_{\mathrm{out}}^{\mathrm{BLR}} \approx 2.19$ is the lowest of the observed objects. 
Table 6. Viewing inclinations, SMBH masses estimates from model (Col. 3), from observations (Col. 4), and from reverberation mapping (Col. 5).

\begin{tabular}{lcccc}
\hline \hline Object & $i\left(^{\circ}\right)$ & $\log \left(M_{\mathrm{MOD}} / M_{\odot}\right)$ & $\log \left(M_{\mathrm{POL}} / M_{\odot}\right)$ & $\log \left(M_{\mathrm{REV}} / M_{\odot}\right)$ \\
\hline \multirow{2}{*}{ NGC 4051 } & 25.01 & $7.2 \pm 0.2$ & & \\
& 32.46 & $6.92 \pm 0.09$ & $6.69 \pm 0.21$ & $6.24 \pm 0.13$ \\
& 38.62 & $6.78 \pm 0.06$ & & \\
NGC 4151 & 25.01 & $7.56 \pm 0.07$ & & $7.12 \pm 0.05$ \\
& 32.46 & $7.40 \pm 0.03$ & $7.21 \pm 0.27$ & \\
& 38.62 & $7.27 \pm 0.04$ & & \\
3C 273 & 25.01 & $8.94 \pm 0.09$ & & \\
& 32.46 & $8.90 \pm 0.09$ & $8.85 \pm 0.27$ & \\
& 38.62 & $8.87 \pm 0.08$ & & $7.85 \pm 0.21$ \\
PG0844+349 & 25.01 & $8.00 \pm 0.08$ & & \\
& 32.46 & $7.95 \pm 0.06$ & $7.70 \pm 0.23$ & \\
\hline
\end{tabular}

Notes. $M_{\mathrm{POL}}$ denotes masses obtained using AP15 which we used as input mass. Masses obtained by reverberation mapping were taken from Bentz \& Katz (2015) using virial factor $\langle f\rangle=4.31 \pm 1.05$ (Grier et al. 2013).

Mass estimates follow the previous trend: the highest estimate for the lowest inclination.

$P G 0844+349$ - We can see from observations that the $\varphi$ profile is asymmetric and that the $\varphi$ amplitude is greater for the red part of the line than for the blue part. The results are similar to those of the first two objects (Fig. A.3), $R_{\text {in }}^{\mathrm{SR}} / R_{\text {out }}^{\mathrm{BLR}} \approx 2.44$.

For all modeled objects, we were able to produce profiles of $\varphi$ that were very similar to the observed profiles (Figs. 12, A.1, A.2, A.3, upper panels). The SMBH masses estimated from the fitting of the model data are higher than those obtained by fitting the observational data, and the obtained values decrease as the viewing inclination increases (Table 6); the $\varphi$ amplitude is very sensitive to inclination and decreases when viewing from face-on towards edge-on inclinations (from lower to greater). For all observed objects, the modeled PO ranges from $0.5 \%$ to $1.5 \%$ for inclinations from lowest to highest. As a measure of the strength of a linear association between the model data and the fit, we give the values of the Pearson correlation coefficient $r$. For all objects we find that the correlation coefficient $r$ is greater than 0.9, except for NGC4151 when viewed from inclination $i=25.01^{\circ}$ (Fig. A.1, lower left panel). The corresponding $p$ values are very close to 0 , indicating a strong linear connection between the modeled data and the fit.

The observational data are much more scattered from the predicted straight line. In general, this yields an error in SMBH estimates that is few times greater than the error obtained by reverberation mapping Afanasiev \& Popović (2015). In the case of NGC 4051 and NGC 4151, modeled data show the best fits for the highest inclination (Figs. 12, A.1, bottom right panels), or is in offset when viewing more towards face-on (Figs. 12, A.1, bottom left and middle panels). The $1 \sigma$ uncertainty is smaller for intermediate inclinations. The largest overestimate of the mass (a factor of 3) is for NGC4051 for $i=25^{\circ}$. For all models $R_{\mathrm{in}}^{\mathrm{SR}} / R_{\mathrm{out}}^{\mathrm{BLR}}>2$. This falls in the regime where the SMBH mass estimation shows dependence on inclination. We achieve the best SMBH mass estimates for inclinations $i \approx 39^{\circ}$, which is close to the value of an average inclination $\left(i=39^{\circ}\right)$ for Type- 1 objects (Lewis et al. 2010; Hryniewicz \& Czerny 2012).

\section{Discussion}

Previous spectropolarimetric studies of Type-1 Seyferts have shown that the polarization signature across the broad $\mathrm{H} \alpha$ varies widely from object to object (Smith et al. 2002). At intermediate viewing inclinations, equatorial scattering dominates the observed polarization and the wavelength averaged polarization $\varphi$ is closely aligned with the projected radio source axis. In their original model, Smith et al. (2005), used a single-scattering approximation, i.e., photons emitted from the BLR are scattered only once from the SR before finally reaching the observer. In their model, the SR is optically thin and we find that an optical depth of at least 1 along with the higher covering factor of the SR is required in order to obtain $\varphi$ and PO comparable with observations.

In our Monte Carlo simulations, the treatment of multiple scattering events was fully performed and we find that the largest fraction of photons is scattered only once, while the other, smaller fraction of photons undergoes a backward scattering from one side of the SR to the other. This secures good circumstances for the application of the AP15 method. In our model, we approximated the emission of an accretion disk as a point source of isotropic continuum emission. We know that this is not the case and that anisotropy arises due to change in the projected surface area and to limb darkening effects (Netzer 1987). The strongest emission is in the direction perpendicular to the disk and rapidly decreases towards edge-on viewing angles. The inner radius of the SR thus cannot be constant, and should follow a dependence on the polar angle that is similar to that of the disk emission (Stalevski et al. 2016). Silicate and graphite dust grains have different sublimation temperatures. Graphite grains can survive up to $\sim 1900 \mathrm{~K}$ and therefore reach closer than silicates which are destroyed when the temperature is $\sim 1200 \mathrm{~K}$. Furthermore, smaller dust grains are destroyed at lower temperatures than the larger grains (Draine 1984; Draine \& Lee 1984; Barvainis 1987). Therefore, we can expect an entire sublimation zone, from graphite to silicate and from larger to smaller dust grains (Kishimoto et al. 2007; Mor \& Netzer 2012). This gives the opportunity for dust particles to inhabit the equatorial region in the close vicinity of the BLR. Equatorial scattering of broad lines from the adjacent SR gives a very low inclination dependence on the parameter $a$ rendering the AP15 method inclination independent.

For SMBH mass estimates using the AP15 method, the inner radius of the torus is needed. It can be obtained directly using dust reverberation in the infrared (Kishimoto et al. 2011). 
The number of objects for which dust reverberation has been performed is smaller than the number of objects for which the reverberation have been performed in the optical. For most of the objects, $R_{\text {in }}^{\text {SR }}$ can be calculated only through scaling relations, which can additionally increase an error in the SMBH estimates. The other way is to calculate $R_{\text {in }}^{\mathrm{SR}}$ from the UV radiation (Barvainis 1987). For this we need to know a priori the physical and chemical composition of dust. Using the right value is important since the estimated $\mathrm{BH}$ mass is directly proportional to the inner radius.

Seyfert 1 galaxies are often highly variable and when they are in a state of minimum activity (up to Type-2), the shape of the position angle of the polarization plane cannot be detected because of the weakness or absence of a flux from the broad line. In this case, the mass estimation using the AP15 method is not applicable, even if the object is confidently assigned to the type of objects with equatorial scattering. Therefore, in future modeling, the variability of an AGN must be taken into account.

Originally the AP15 method was proposed for systems with an inclination between $20^{\circ}$ and $70^{\circ}$. For high viewing inclinations, we have Type- 2 objects for which polar scattering dominates the polarization signal and the method is no longer valid. For almost pole-on AGN, the AP15 method faces two problems. First, the amount of interstellar polarization can dominate over the amount of scattering-induced radiation from the innermost regions of AGN. The amount of interstellar polarization is wavelength-dependent and is often maximum in the optical band (see Serkowski et al. 1975). Since the polarization signal of polar AGN in the optical band is usually much lower than $1 \%$ in the optical (Smith et al. 2002; Marin 2014), the AP15 method is thus restricted to inclinations higher than $20^{\circ}$. Second, the method overestimates the SMBH mass by a factor of 1.5 in comparison with the value obtained for the lowest inclination when $R_{\text {in }}^{\mathrm{SR}} / R_{\text {out }}^{\mathrm{BLR}} \approx 2$. When the SR is closer, the inclination effect is lower and the mass estimates only depend on the SR inner radius.

It is important to note here that several recent works (Piotrovich et al. 2015; Baldi et al. 2016; Songsheng \& Wang 2018) give some ideas for using spectropolarimetry to estimate BH mass in AGNs. Basically, all these papers try to constrain the virial factor (Piotrovich et al. 2015; Songsheng \& Wang 2018) or to use the broad polarized line (Baldi et al. 2016) to find the black hole mass. In the work by Songsheng \& Wang (2018), the authors performed Monte Carlo simulations for a wide range of parameters assuming a static flared-disk geometry for the equatorial region. In comparison to the unpolarized spectra, the virial factor of the polarized spectra has a much narrower distribution. Furthermore, the half-opening angle of the BLR and the nucleus inclinations appear to be the two parameters with the highest influence on the virial factor. The difference between the methods mentioned above and AP15 is that the AP15 method provides a direct measurement of the $\mathrm{BH}$ mass from the polarization angle, and here we also used some approaches similar to those used in Songsheng \& Wang (2018), but focusing on a polarization plane position angle $\varphi$ and the limits of the AP15 method. In comparison, our 3D polarized radiative transfer simulations have shown that the polarization plane position angle is largely affected by the distance between the BLR and SR. If the two share similar values, the mass estimated using the AP15 method becomes inclination independent, which is a great advantage in comparison to traditional reverberation mapping techniques.

\section{Conclusions}

We modeled polarization effects in AGN broad lines in order to constrain the limits of the AP15 method for the BH estimates using polarization in broad lines.

We used the Monte Carlo radiative transfer code stokes, which includes multiple scattering for accurate polarization treatment. We considered equatorial scattering (on the torus) of the light from a BLR that has dominant Keplerian motion. Additionally, we considered complex BLR kinematics having inflows and outflows.

We explored all these effects on the accuracy of the BH mass measurement using the AP15 method.

From our investigation we can outline following conclusions:

- If Keplerian motion can be traced through the polarized line profile, then direct estimates of the mass can be performed to obtain reasonable values.

- The effects of possible inflow/outflow configuration of the BLR take its toll only for extreme cases where the velocity of the inflowing/outflowing emitter is comparable to or higher than the Keplerian velocity.

- Masses of the SMBHs obtained by the AP15 method are in good agreement with those found in the literature.

The AP15 method gives us a new independent way of mass estimation. Future parameter grids will be extended with the inflow/outflow configuration of the scattering region with the possibility of considering clumpy structures. We expect to perform high-quality spectropolarimetric observations of the highredshifted quasars and to test the AP15 method on highly ionized lines such as C III and C IV.

Acknowledgements. We thank an anonymous referee for the constructive suggestions that improved this paper. This work was supported by the Ministry of Education and Science (Republic of Serbia) through the project Astrophysical Spectroscopy of Extragalactic Objects (176001), the French PNHE and the grant ANR-11-JS56-013-01 "POLIOPTIX", and the Russian Foundation for Basic Research grant N15-02-02101 and N14-22-03006. D. Savić thanks the French Government and the French Embassy in Serbia for supporting his research. Part of this work was supported by the COST Action MP1104 "Polarization as a tool to study the solar system and beyond".

\section{References}

Afanasiev, V. L., \& Amirkhanyan, V. R. 2012, Astrophys. Bull., 67, 438

Afanasiev, V. L., \& Moiseev, A. V. 2005, Astron. Lett., 31, 194

Afanasiev, V. L., \& Moiseev, A. V. 2011, Baltic Astron., 20, 363

Afanasiev, V. L., \& Popović, L.Č. 2015, ApJ, 800, L35

Afanasiev, V. L., Borisov, N. V., Gnedin, Y. N., et al. 2011, Astron. Lett., 37, 302 Afanasiev, V. L., Popović, L. Č., Shapovalova, A. I., Borisov, N. V., \& Ilić, D. 2014, MNRAS, 440, 519

Antonucci, R. 1993, ARA\&A, 31, 473

Appenzeller, I. 1968, ApJ, 151, 769

Baldi, R. D., Capetti, A., Robinson, A., Laor, A., \& Behar, E. 2016, MNRAS, 458, L69

Barvainis, R. 1987, ApJ, 320, 537

Bentz, M. C., \& Katz, S. 2015, PASP, 127, 67

Bentz, M. C., Denney, K. D., Cackett, E. M., et al. 2006, ApJ, 651, 775

Bentz, M. C., Denney, K. D., Grier, C. J., et al. 2013, ApJ, 767, 149

Blandford, R. D., \& McKee, C. F. 1982, ApJ, 255, 419

Boller, T. 2001, in New Century of X-ray Astronomy, eds. H. Inoue \& H. Kunieda, ASP Conf. Ser., 251, 112

Courvoisier, T. J.-L., Turner, M. J. L., Robson, E. I., et al. 1987, A\&A, 176, 197 Courvoisier, T. J. L., Robson, E. I., Blecha, A., et al. 1990, A\&A, 234, 73

de Vaucouleurs, G., de Vaucouleurs, A., Corwin, Jr. H. G., et al. 1991, S\&T, 82, 621

Draine, B. T. 1984, ApJ, 277, L71

Draine, B. T., \& Lee, H. M. 1984, ApJ, 285, 89

Fabian, A. C. 2012, ARA\&A, 50, 455

Ferrarese, L., \& Ford, H. 2005, Space Sci. Rev., 116, 523

Ferrarese, L., Pogge, R. W., Peterson, B. M., et al. 2001, ApJ, 555, L79 
Gaskell, C. M. 2009, New Astron. Rev., 53, 140

Gebhardt, K., Bender, R., Bower, G., et al. 2000, ApJ, 539, L13

Genzel, R., Eisenhauer, F., \& Gillessen, S. 2010, Rev. Mod. Phys., 82, 3121

Goosmann, R. W., \& Gaskell, C. M. 2007, A\&A, 465, 129

Grier, C. J., Peterson, B. M., Pogge, R. W., et al. 2012, ApJ, 755, 60

Grier, C. J., Martini, P., Watson, L. C., et al. 2013, ApJ, 773, 90

Gu, M., Cao, X., \& Jiang, D. R. 2001, MNRAS, 327, 1111

Heckman, T. M., \& Best, P. N. 2014, ARA\&A, 52, 589

Hryniewicz, K., \& Czerny, B. 2012, Mem. Soc. Astron. It., 83, 146

Hutsemékers, D., Agís González, B., Sluse, D., Ramos Almeida, C., \& Acosta Pulido J.-A. 2017, A\&A, 604, L3

Kaspi, S., Smith, P. S., Netzer, H., et al. 2000, ApJ, 533, 631

Kaspi, S., Maoz, D., Netzer, H., et al. 2005, ApJ, 629, 61

Kellermann, K. I., Sramek, R. A., Schmidt, M., Green, R. F., \& Shaffer, D. B. 1994, AJ, 108, 1163

Kishimoto, M., Hönig, S. F., Beckert, T., \& Weigelt, G. 2007, A\&A, 476, 713

Kishimoto, M., Hönig, S. F., Antonucci, R., et al. 2011, A\&A, 536, A78

Kormendy, J., \& Ho, L. C. 2013, ARA\&A, 51, 511

Kormendy, J., \& Richstone, D. 1995, ARA\&A, 33, 581

Koshida, S., Minezaki, T., Yoshii, Y., et al. 2014, ApJ, 788, 159

Krolik, J. H., \& Begelman, M. C. 1988, ApJ, 329, 702

Laor, A. 2000, ApJ, 543, L111

Lewis, K. T., Eracleous, M., \& Storchi-Bergmann, T. 2010, ApJS, 187, 416

Lynden-Bell, D. 1969, Nature, 223, 690

Marin, F. 2014, MNRAS, 441, 551

Marin, F. 2017, A\&A, 607, A40

Marin, F., \& Goosmann, R. W. 2014, in SF2A-2014: Proceedings of the Annual Meeting of the French Society of Astronomy and Astrophysics, eds. J. Ballet, F. Martins, F. Bournaud, R. Monier, \& C. Reylé, 103

Marin, F., Goosmann, R. W., Gaskell, C. M., Porquet, D., \& Dovčiak M. 2012, A\&A, 548, A121

Marin, F., Goosmann, R. W. \& Gaskell, C. M. 2015, A\&A, 577, A66

Marin, F., Goosmann, R. W., \& Petrucci, P.-O. 2016, A\&A, 591, A23
Martin, P. G., Thompson, I. B., Maza, J., \& Angel, J. R. P. 1983, ApJ, 266, 470 McHardy, I. M., Papadakis, I. E., Uttley, P., Page, M. J., \& Mason, K. O. 2004, MNRAS, 348, 783

Meyer, L., Ghez, A. M., Schödel, R., et al. 2012, Science, 338, 84

Miyoshi, M., Moran, J., Herrnstein, J., et al. 1995, Nature, 373, 127

Mor, R., \& Netzer, H. 2012, MNRAS, 420, 526

Netzer, H. 1987, MNRAS, 225, 55

Netzer, H. 2013, The Physics and Evolution of Active Galactic Nuclei (Cambridge, UK: Cambridge University Press)

Netzer, H., \& Laor, A. 1993, ApJ, 404, L51

Penston, M. V., \& Perez, E. 1984, MNRAS, 211, 33

Peterson, B. M. 2014, Space Sci. Rev., 183, 253

Peterson, B. M., McHardy, I. M., Wilkes, B. J., et al. 2000, ApJ, 542, 161

Peterson, B. M., Ferrarese, L., Gilbert, K. M., et al. 2004, ApJ, 613, 682

Peterson, B. M., Denney, K. D., De Rosa, G., et al. 2013, ApJ, 779, 109

Piotrovich, M. Y., Gnedin, Y. N., Silant'ev, N. A., Natsvlishvili, T. M., \& Buliga, S. D. 2015, MNRAS, 454, 1157

Runnoe, J. C., Brotherton, M. S., \& Shang, Z. 2012, MNRAS, 427, 1800

Schmidt, M. 1963, Nature, 197, 1040

Schmidt, M., \& Green, R. F. 1983, ApJ, 269, 352

Serkowski, K., Mathewson, D. S., \& Ford, V. L. 1975, ApJ, 196, 261

Shapovalova, A. I., Popović, L. Č., Collin, S., et al. 2008, A\&A, 486, 99

Shapovalova, A. I., Popović, L. Č., Burenkov, A. N., et al. 2010, A\&A, 509, A106

Smith, J. E., Young, S., Robinson, A., et al. 2002, MNRAS, 335, 773

Smith, J. E., Robinson, A., Young, S., Axon, D. J., \& Corbett, E. A. 2005, MNRAS, 359, 846

Songsheng, Y.-Y., \& Wang, J.-M. 2018, MNRAS, 473, L1

Stalevski, M., Ricci, C., Ueda, Y., et al. 2016, MNRAS, 458, 2288

Thomson, R. C., Mackay, C. D., \& Wright, A. E. 1993, Nature, 365, 133

Urry, C. M., \& Padovani, P. 1995, PASP, 107, 803

Whiteoak, J. B. 1966, Z. Astrophys., 64, 181

Woo, J.-H., \& Urry, C. M. 2002, ApJ, 579, 530

Zu, Y., Kochanek, C. S., \& Peterson, B. M. 2011, ApJ, 735, 80 
Đ. Savić et al.: AGN black hole mass estimates using polarization in broad emission lines

\section{Appendix A: Additional figures}
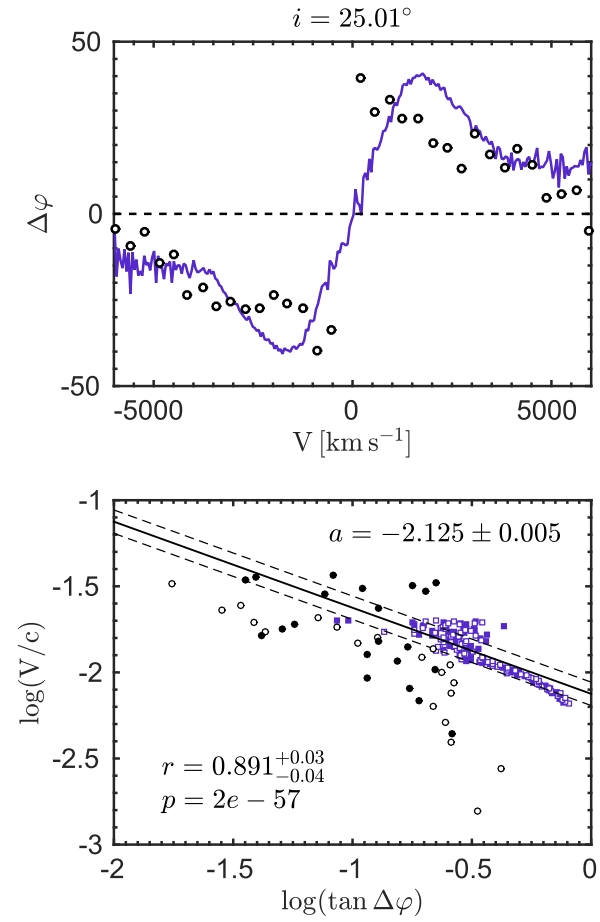

Fig. A.1. Same as in Fig. 12, but for NGC4151.
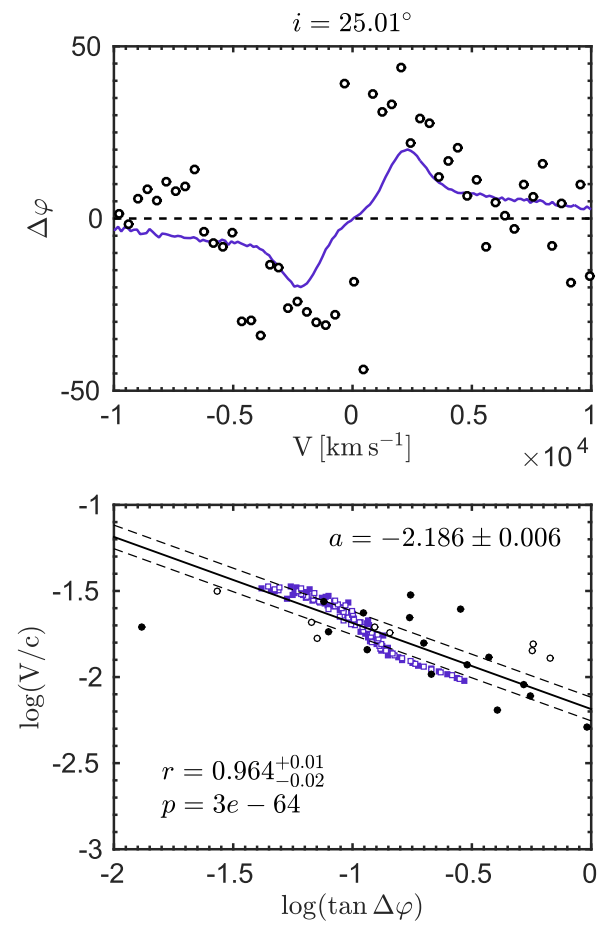
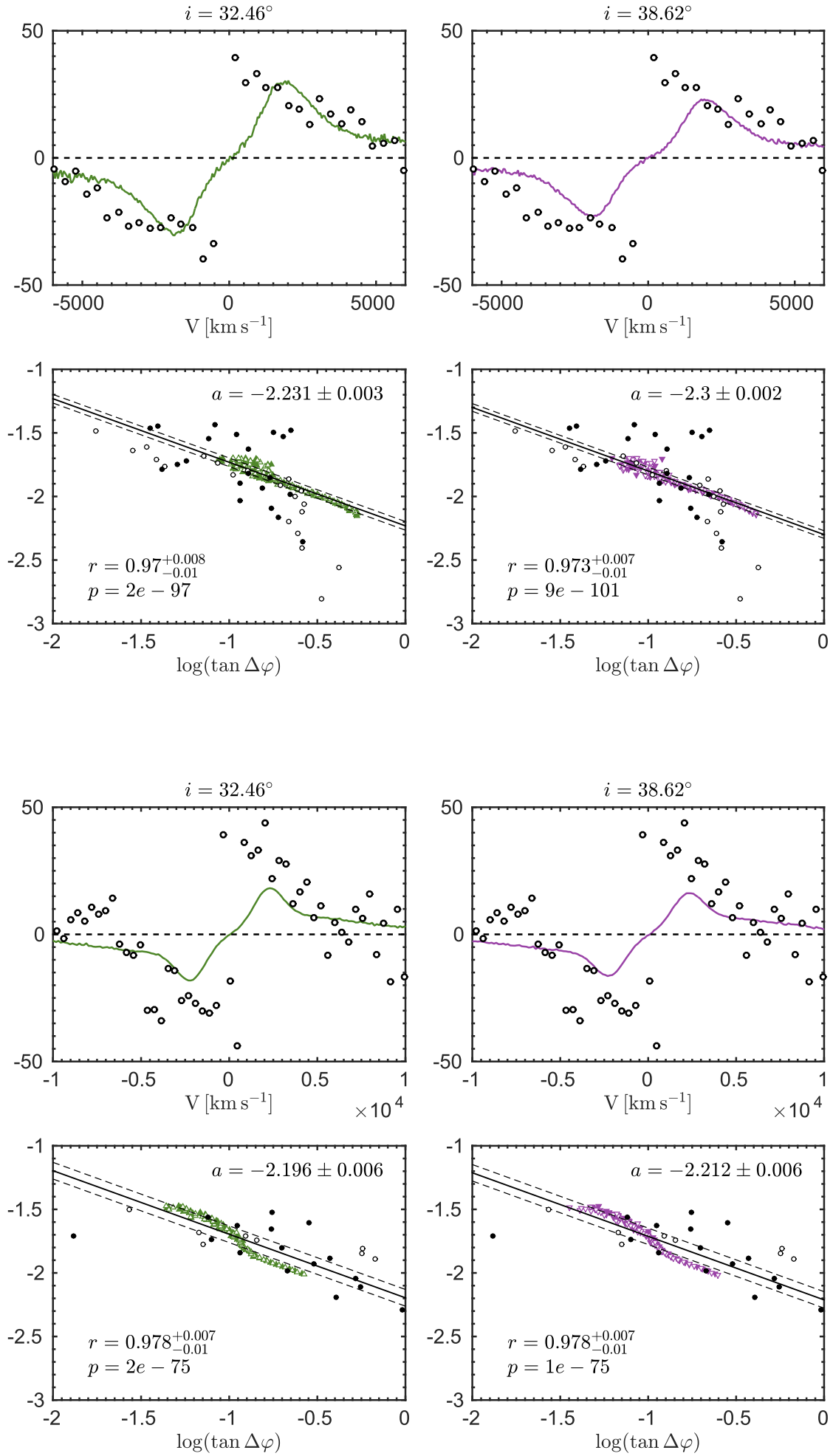

Fig. A.2. Same as in Fig. 12, but for 3C273. 
A\&A 614, A120 (2018)
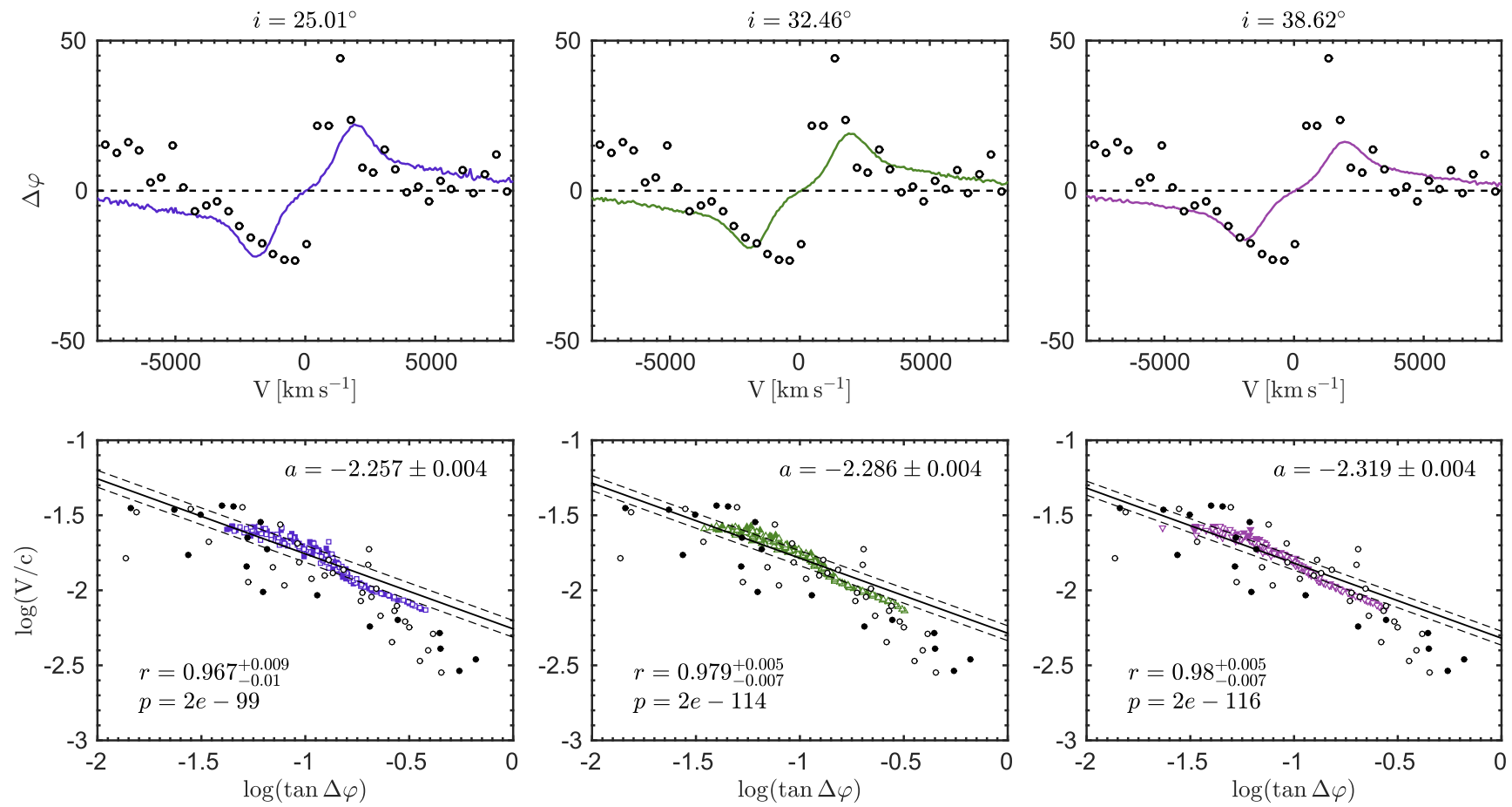

Fig. A.3. Same as in Fig. 12, but for PG0844+349. 